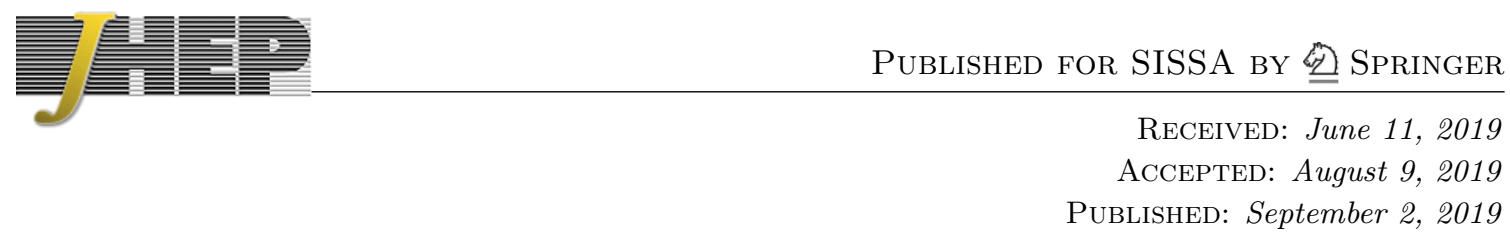

\title{
Vacuum instabilities in the N2HDM
}

\section{P.M. Ferreira, ${ }^{a, b}$ Rui Santos, ${ }^{a, b}$ Margarete Mühlleitner, ${ }^{c}$ Georg Weiglein ${ }^{d}$ and Jonas Wittbrodt ${ }^{d}$}

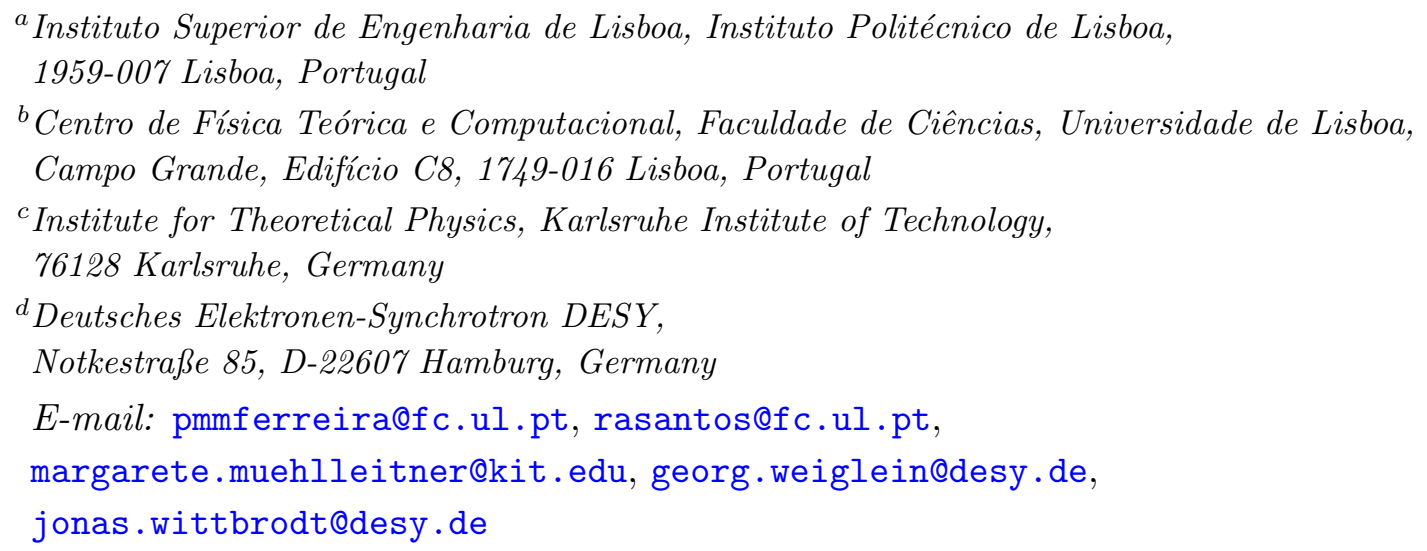

Abstract: The Higgs sector of the Next-to-Minimal Two-Higgs-Doublet Model (N2HDM) is obtained from the Two-Higgs-Doublet Model (2HDM) containing two complex Higgs doublets, by adding a real singlet field. In this paper, we analyse the vacuum structure of the N2HDM with respect to the possibility of vacuum instabilities. We show that while one type of charge- and CP-preserving vacuum cannot coexist with deeper chargeor CP-breaking minima, there is another type of vacuum whose stability is endangered by the possible occurrence of deeper charge- and CP-breaking minima. Analytical expressions relating the depth of different vacua are deduced. Parameter scans of the model are carried out that illustrate the regions of parameter space where the vacuum is either stable or metastable as well as the regions where tunnelling to deeper vacua gives rise to a too short lifetime of the vacuum. Taking other experimental and theoretical constraints into account, we find that the vacuum stability constraints have an important impact on the phenomenology of the N2HDM.

Keywords: Beyond Standard Model, Higgs Physics

ARXIV EPRINT: 1905.10234 


\section{Contents}

1 Introduction 1

2 The model and possible minima 3

$\begin{array}{lll}2.1 & \text { Stability of normal minima against charge breaking } & 7\end{array}$

2.1.1 Extrema $\mathcal{N}$ vs. $\mathcal{C B}$ and $\mathcal{C B} s \quad 8$

$\begin{array}{lll}2.1 .2 & \text { Extrema } \mathcal{N} s \text { vs. } \mathcal{C B} \text { and } \mathcal{C B} s & 10\end{array}$

2.2 Stability of normal minima against CP breaking 11

$\begin{array}{lll}2.2 .1 & \text { Extrema } \mathcal{N} \text { vs. } \mathcal{C P} \text { and } \mathcal{C P} s & 12\end{array}$

2.2.2 Extrema $\mathcal{N} s$ vs. $\mathcal{C P}$ and $\mathcal{C P} s \quad 13$

$\begin{array}{lll}2.3 & \text { Other coexisting neutral minima } & 14\end{array}$

$\begin{array}{lll}2.4 \text { Vacuum stability } & 15\end{array}$

3 Numerical analysis $\quad 16$

$\begin{array}{lll}3.1 & \text { Parameter scan } & 16\end{array}$

$\begin{array}{lll}3.2 & \text { Numerical vacuum stability } & 18\end{array}$

$\begin{array}{lll}3.3 & \text { Discussion } & 19\end{array}$

4 Conclusions $\quad 25$

$\begin{array}{ll}\text { A On the nature of stationary points } & 26\end{array}$

\section{Introduction}

The Higgs mechanism [1-5] has been introduced to generate particle masses without violating gauge symmetries. It is based on a sufficiently stable vacuum with non-zero vacuum expectation value $v \approx 246 \mathrm{GeV}$. Within the Standard Model (SM), the stability of the electroweak $(\mathrm{EW})$ vacuum is guaranteed at lowest order as a consequence of the postulated form of the Higgs potential. Through higher-order corrections, the stability of the EW vacuum becomes intimately related also to the other Standard Model (SM) parameters [6, 7], in particular the top quark mass. When extrapolating the SM to high energy scales it turns out that the EW vacuum is metastable for scales larger than about $10^{10} \mathrm{GeV}$, which means that for these scales the vacuum is no longer absolutely stable but has a lifetime that is longer than the age of the universe.

Extensions beyond the SM (BSM) typically introduce new additional scalar degrees of freedom. While the loop contributions of these scalar particles may counteract the impact of the top quark loop, the presence of the additional scalars modifies the structure of the Higgs potential such that additional vacua can occur that are different from the one related to the correct EW symmetry breaking (EWSB). There can be vacua that break 
the CP symmetry (CP breaking) or the conservation of electric charge (charge breaking), in supersymmetric models even color breaking minima can occur. Moreover, there is the possibility of a second EW minimum but with a wrong vacuum expectation value (VEV) $v \neq 246 \mathrm{GeV}$, as for example in the 2-Higgs Doublet Model (2HDM) where this situation was named "panic vacuum" [8-12]. In case an additional vacuum is deeper than the EW one then tunnelling can occur into dangerous non-physical vacuum configurations [13]. If this happens at time scales beyond the lifetime of the universe the EW vacuum is considered as metastable. However, parameter regions giving rise to faster vacuum decays are regarded as unphysical and should be excluded. Hence the requirement of a stable EW vacuum at cosmological time scales has immediate consequences for the allowed parameter space of the models. A thorough analysis of their vacuum structure is therefore crucial to correctly identify the allowed parameter space and consequently make appropriate predictions for observables and signatures for the experimental studies.

The analysis of the $2 \mathrm{HDM}[8,9,14,15]$ has shown that if a "normal" vacuum exists, i.e. a vacuum that is EW breaking but charge and CP conserving, any stationary point that is charge or CP breaking is necessarily a saddle point that lies above the normal minimum. There is also the possibility to have a second normal minimum but with the wrong VEV, i.e. a panic vacuum state. The Inert $2 \mathrm{HDM}$, a $2 \mathrm{HDM}$ with an exact $\mathbb{Z}_{2}$ symmetry, can have two types - Inert and Inert-like - of minima which can coexist with one another for certain parameter relations. The one-loop study, however, has shown [16] that the parameter regions where this is the case can change at loop level. The analysis of the possibility of a strong first order phase transition in the context of a CP-conserving and $\mathrm{CP}$-violating $2 \mathrm{HDM}$ conducted in $[17,18]$ revealed as a side product that the allowed minima at leading order do not necessarily lead to stable physical configurations at nextto-leading order (NLO) and vice versa. The developed code BSMPT [19] allows for studies of the vacuum structure at NLO (at zero and at finite temperature) of arbitrary user-defined BSM extensions. This is also the case for Vevacious [20,21], designed for general BSM models, including one-loop and temperature effects. Recently, members of this collaboration have presented an approach at leading order for an efficient and reliable evaluation of the constraints from vacuum stability and applied it to the minimal supersymmetric extension of the SM (MSSM) [22]. As shown in [23, 24] and also discussed in [22], for calculations of the vacuum decay lifetime the loop-corrected effective potential in general does not correspond to a consistent perturbative expansion. A first analysis of the vacuum structure of the N2HDM has been carried out by some of the present authors in [25]. The N2HDM, which is obtained upon extension of the 2HDM with a real singlet field (which may acquire a VEV), was shown to exhibit a different vacuum structure than the 2HDM. Thus e.g. charge or CP breaking minima deeper than the normal minimum can exist.

In this paper we perform a detailed analysis of the vacuum structure of the N2HDM. We classify the different possible types of vacua and derive analytical expressions for the comparison of the values of the potential at minima of different nature. In contrast to [25], where a general phenomenological analysis of the N2HDM was performed (and where parts of our results have been presented in a numerical approach), we concentrate here on the vacuum structure itself and its implications on the model. By applying the method of [22], 
we investigate the requirement of a sufficiently stable physical minimum on the allowed parameter range. In particular, we investigate here for the first time the impact of the N2HDM vacuum structure on the phenomenology of the model. Moreover, we discuss the importance of including parameter regions with a metastable vacuum in phenomenological analyses in order to avoid incorrect conclusions on the viability of parameter space regions. Our study thus makes important new contributions to properly constraining the viable parameter space taking into account the theoretical constraints from the requirement of a stable vacuum.

The paper is organised as follows. In section 2 we introduce the model and the different types of possible minima. It contains the detailed analytical analysis of the stability of the different minima. Section 3 is dedicated to the numerical analysis of the vacuum structure of the N2HDM. We describe our parameter scan and the method that we apply in order to identify the regions where the vacuum is stable or metastable. Subsequently we present and discuss our numerical results. We conclude in section 4. The appendix contains a derivation that is used in our determination of the nature of the stationary points.

\section{The model and possible minima}

The particle content of the N2HDM is identical to the one of the 2HDM in the fermionic and gauge sectors, but includes an extra real singlet scalar field, $\Phi_{S}$. To reduce the large number of parameters of the scalar potential, and to allow for the possibility of interesting phenomenology, such as dark matter, three discrete symmetries are imposed: (a) a $\mathbb{Z}_{2}$ symmetry in which one of the doublets is affected by a sign change, $\Phi_{1} \rightarrow \Phi_{1}, \Phi_{2} \rightarrow-\Phi_{2}$ and $\Phi_{S} \rightarrow \Phi_{S}$; (b) another $\mathbb{Z}_{2}$ symmetry which leaves the doublets unchanged but changes the sign of the singlet, $\Phi_{1} \rightarrow \Phi_{1}, \Phi_{2} \rightarrow \Phi_{2}$ and $\Phi_{S} \rightarrow-\Phi_{S}$; (c) the standard CP symmetry, $\Phi_{1} \rightarrow \Phi_{1}^{*}$ and $\Phi_{2} \rightarrow \Phi_{2}^{*}$ - since the singlet is real, the CP transformation does not affect it. After imposing these symmetries only terms quadratic and quartic in the fields are allowed and the most general scalar potential is given by

$$
\begin{aligned}
V= & m_{11}^{2}\left|\Phi_{1}\right|^{2}+m_{22}^{2}\left|\Phi_{2}\right|^{2}-\left(m_{12}^{2} \Phi_{1}^{\dagger} \Phi_{2}+\text { h.c. }\right) \\
& +\frac{1}{2} \lambda_{1}\left|\Phi_{1}\right|^{4}+\frac{1}{2} \lambda_{2}\left|\Phi_{2}\right|^{4}+\lambda_{3}\left|\Phi_{1}\right|^{2}\left|\Phi_{2}\right|^{2}+\lambda_{4}\left|\Phi_{1}^{\dagger} \Phi_{2}\right|^{2}+\frac{1}{2} \lambda_{5}\left[\left(\Phi_{1}^{\dagger} \Phi_{2}\right)^{2}+\text { h.c. }\right] \\
& +\frac{1}{2} m_{S}^{2} \Phi_{S}^{2}+\frac{1}{8} \lambda_{6} \Phi_{S}^{4}+\frac{1}{2} \lambda_{7}\left|\Phi_{1}\right|^{2} \Phi_{S}^{2}+\frac{1}{2} \lambda_{8}\left|\Phi_{2}\right|^{2} \Phi_{S}^{2},
\end{aligned}
$$

where all parameters in the potential are real. We allow for the $\mathbb{Z}_{2}$ symmetry (a) to be softly broken by the $m_{12}^{2}$ term. The theory obviously also includes fermions, and the Yukawa Lagrangian will depend on the choices made to extend the discrete symmetries imposed upon the scalar sector to the fermion one. Due to gauge invariance the singlet field $\Phi_{S}$ couples to neither fermions nor gauge bosons. Therefore, the Yukawa Lagrangian will have four different possible forms, identical to the different types of 2HDM Yukawa Lagrangians. All of the four different possibilities lead to flavour conservation in scalar interactions. One of the possibilities (achieved if all right fermion fields change sign under 
the first $\mathbb{Z}_{2}$ symmetry defined above) is a Type-I model, in which all fermions only couple to the doublet $\Phi_{2}$, and the Yukawa Lagrangian for the third generation is given by

$$
-\mathcal{L}_{Y}=\lambda_{t} \bar{Q}_{L} \tilde{\Phi}_{2} t_{R}+\lambda_{b} \bar{Q}_{L} \Phi_{2} b_{R}+\lambda_{\tau} \bar{L}_{L} \Phi_{2} \tau_{R}
$$

with $Q_{L}$ and $L_{L}$ denoting the left-handed quark and lepton doublets, and $t_{R}, b_{R}$ and $\tau_{R}$ the right-handed top, bottom and tau singlets. The remaining three Yukawa types can be defined analogously [25].

The N2HDM contains different phases, depending on the type of symmetry breaking that occurs. Vacuum expectation values for the scalar fields will lead to vacua which may, or may not, preserve the symmetries imposed. Let us now review the different types of vacua possible in the N2HDM. For the purpose of studying the interplay between different possible vacua, it is convenient to introduce a bilinear formalism, similar to that which has been developed for the 2HDM [8-10, 14, 15, 26-34]. This formalism has been applied to models with different scalar content, for instance the $3 \mathrm{HDM}[35,36]$ or the complex singlet-doublet model [37]. For the N2HDM let us define five real quantities,

$$
x_{1}=\left|\Phi_{1}\right|^{2}, x_{2}=\left|\Phi_{2}\right|^{2}, x_{3}=\operatorname{Re}\left(\Phi_{1}^{\dagger} \Phi_{2}\right), x_{4}=\operatorname{Im}\left(\Phi_{1}^{\dagger} \Phi_{2}\right), x_{5}=\frac{1}{2} \Phi_{S}^{2} .
$$

Further, we define the vectors $X, A$ and the symmetric matrix $B$ as

$$
X=\left(\begin{array}{l}
x_{1} \\
x_{2} \\
x_{3} \\
x_{4} \\
x_{5}
\end{array}\right), \quad A=\left(\begin{array}{c}
m_{11}^{2} \\
m_{22}^{2} \\
-2 m_{12}^{2} \\
0 \\
m_{S}^{2}
\end{array}\right), \quad B=\left(\begin{array}{ccccc}
\lambda_{1} & \lambda_{3} & 0 & 0 & \lambda_{7} \\
\lambda_{3} & \lambda_{2} & 0 & 0 & \lambda_{8} \\
0 & 0 & 2\left(\lambda_{4}+\lambda_{5}\right) & 0 & 0 \\
0 & 0 & 0 & 2\left(\lambda_{4}-\lambda_{5}\right) & 0 \\
\lambda_{7} & \lambda_{8} & 0 & 0 & \lambda_{6}
\end{array}\right),
$$

in terms of which the potential of eq. (2.1) can be rewritten as

$$
V=A^{T} X+\frac{1}{2} X^{T} B X .
$$

In what follows we shall also make extensive use of the vector

$$
V^{\prime}=\frac{\partial V}{\partial X^{T}}=A+B X .
$$

It can easily be shown that, at a given stationary point in which the fields acquire vacuum expectation values such that $\langle X\rangle^{T}=\left(\left\langle\left|\Phi_{1}\right|\right\rangle^{2},\left\langle\left|\Phi_{2}\right|\right\rangle^{2}, \operatorname{Re}\left\langle\Phi_{1}^{\dagger} \Phi_{2}\right\rangle, \operatorname{Im}\left\langle\Phi_{1}^{\dagger} \Phi_{2}\right\rangle, \frac{1}{2}\left\langle\Phi_{S}\right\rangle^{2}\right)^{T}$, the value of the potential at that stationary point, $V_{S P}$, is given by

$$
V_{S P}=\frac{1}{2} A^{T}\langle X\rangle=-\frac{1}{2}\langle X\rangle^{T} B\langle X\rangle .
$$

As explained in [25], by using the gauge freedom of the model, it is always possible to bring the most generic possible vacuum (in which, in principle, one would have nine different VEVs to consider, since the scalar doublets and singlet have a total of nine real component fields) to a simple form, to wit

$$
\left\langle\Phi_{1}\right\rangle=\frac{1}{\sqrt{2}}\left(\begin{array}{c}
0 \\
v_{1}
\end{array}\right), \quad\left\langle\Phi_{2}\right\rangle=\frac{1}{\sqrt{2}}\left(\begin{array}{c}
v_{\mathrm{cb}} \\
v_{2}+\mathrm{i} v_{\mathrm{cp}}
\end{array}\right), \quad\left\langle\Phi_{S}\right\rangle=v_{S},
$$


where all VEVs $v_{X}$ are, without loss of generality, real. The charge breaking VEV $v_{\mathrm{cb}}$ breaks electromagnetic symmetry (giving the photon a mass) and the VEV $v_{\mathrm{cp}}$ breaks $\mathrm{CP}$ conservation. It is easy to verify that these VEVs cannot coexist simultaneously. In other words, the minimisation of the potential implies that, if $v_{\mathrm{cb}} \neq 0$ then $v_{\mathrm{cp}}=0$, and vice-versa.

Different non-zero VEVs lead to different types of symmetry breaking, originating from minima which preserve, or not, distinct symmetries. The classification of all possible vacua was first made in [25], but here we adopt a different notation better suited for our analysis. There are two possible charge charge breaking vacua; two $\mathrm{CP}$ breaking vacua; two normal (electroweak breaking, but charge and CP conserving) vacua; and a single vacuum for which electroweak symmetry is unbroken. ${ }^{1}$ Thus a total of seven possible types of vacua, or phases, exists in the model. The two electroweak breaking but charge and CP conserving vacua of the N2HDM most closely resemble a SM-like vacuum, in that they have a CP-even scalar field which can mimic the SM Higgs boson. However, the N2HDM involves extra scalars, including a charged one and several neutral ones with definite CP quantum numbers, and possibly a dark matter candidate.

The first normal stationary point $\mathcal{N}$ (denoted $I$ in [25]) occurs when the parameters of the potential are such that the minimisation conditions of the potential allow a solution for which both doublets have neutral, real VEVs and the singlet has none. This vacuum therefore preserves the $\mathbb{Z}_{2}$ symmetry of the singlet - the singlet has no VEV and does not mix with the remaining neutral scalars. Hence this corresponds to the dark matter phase of the model, with the VEVs

$$
\left\langle\Phi_{1}\right\rangle_{\mathcal{N}}=\frac{1}{\sqrt{2}}\left(\begin{array}{c}
0 \\
v_{1}
\end{array}\right), \quad\left\langle\Phi_{2}\right\rangle_{\mathcal{N}}=\frac{1}{\sqrt{2}}\left(\begin{array}{c}
0 \\
v_{2}
\end{array}\right), \quad\left\langle\Phi_{S}\right\rangle_{\mathcal{N}}=0 .
$$

This results in the following values for the $X$ and $V^{\prime}$ vectors (defined in eqs. (2.4) and (2.6)):

$$
X_{\mathcal{N}}=\langle X\rangle_{\mathcal{N}}=\frac{1}{2}\left(\begin{array}{c}
v_{1}^{2} \\
v_{2}^{2} \\
v_{1} v_{2} \\
0 \\
0
\end{array}\right), \quad V_{\mathcal{N}}^{\prime}=A+B X_{\mathcal{N}}=\left(\begin{array}{c}
\frac{v_{2}^{2}}{v^{2}} m_{H^{ \pm}}^{2} \\
\frac{v_{1}^{2}}{v^{2}} m_{H^{ \pm}}^{2} \\
-\frac{2 v_{1} v_{2}}{v^{2}} m_{H^{ \pm}}^{2} \\
0 \\
m_{D}^{2}
\end{array}\right)
$$

with $v^{2}=v_{1}^{2}+v_{2}^{2}$. The entries of $V_{\mathcal{N}}^{\prime}$ are dictated by the $\mathcal{N}$ minimisation conditions and by the respective eigenvalues of the scalar mass matrices, where $m_{H^{ \pm}}^{2}$ is the squared charged scalar mass at this stationary point and $m_{D}^{2}$ the squared mass of the singlet field. These are given by

$$
m_{H^{ \pm}}^{2}=m_{12}^{2} \frac{v^{2}}{v_{1} v_{2}}-\frac{1}{2}\left(\lambda_{4}+\lambda_{5}\right) v^{2}, \quad m_{D}^{2}=m_{S}^{2}+\frac{1}{2}\left(\lambda_{7} v_{1}^{2}+\lambda_{8} v_{2}^{2}\right) .
$$

Using eq. (2.7) the value of the potential at this stationary point may be written as

$$
V_{\mathcal{N}}=\frac{1}{2} A^{T} X_{\mathcal{N}}=-\frac{1}{2} X_{\mathcal{N}}^{T} B X_{\mathcal{N}}
$$

\footnotetext{
${ }^{1}$ We exclude, from this list, the trivial extremum at the origin, in which no field acquires a VEV.
} 
The second normal stationary point $\mathcal{N} s$ (denoted $s I$ in [25]) corresponds to a solution of the minimisation conditions where both the doublets and the singlet $\Phi_{S}$ acquire non-zero VEVs. This additionally breaks the singlet $\mathbb{Z}_{2}$ symmetry — thus the singlet $\Phi_{S}$ will mix with the remaining neutral scalars. Starting from the following VEV configuration

$$
\left\langle\Phi_{1}\right\rangle_{\mathcal{N} s}=\frac{1}{\sqrt{2}}\left(\begin{array}{c}
0 \\
v_{1}^{\prime}
\end{array}\right), \quad\left\langle\Phi_{2}\right\rangle_{\mathcal{N} s}=\frac{1}{\sqrt{2}}\left(\begin{array}{c}
0 \\
v_{2}^{\prime}
\end{array}\right), \quad\left\langle\Phi_{S}\right\rangle_{\mathcal{N} s}=v_{S}^{\prime},
$$

we define

$$
X_{\mathcal{N} s}=\langle X\rangle_{\mathcal{N} s}=\frac{1}{2}\left(\begin{array}{c}
v_{1}^{\prime 2} \\
v_{2}^{\prime 2} \\
v_{1}^{\prime} v_{2}^{\prime} \\
0 \\
v_{s}^{\prime 2}
\end{array}\right) \quad, \quad V_{\mathcal{N} s}^{\prime}=A+B X_{\mathcal{N} s}=\left(m_{H^{ \pm}}^{2}\right)_{\mathcal{N} s}\left(\begin{array}{c}
\frac{v_{2}^{\prime 2}}{v^{\prime 2}} \\
\frac{v_{1}^{\prime 2}}{v^{\prime 2}} \\
-\frac{2 v_{1}^{\prime} v_{2}^{\prime}}{v^{\prime 2}} \\
0 \\
0
\end{array}\right)
$$

where ${v^{\prime}}^{2}=v_{1}^{\prime 2}+v_{2}^{\prime 2}$ and $\left(m_{H^{ \pm}}^{2}\right)_{\mathcal{N} s}$ is the squared charged scalar mass at the $\mathcal{N} s$ stationary point, given by

$$
\left(m_{H^{ \pm}}^{2}\right)_{\mathcal{N} s}=m_{12}^{2} \frac{v^{\prime 2}}{v_{1}^{\prime} v_{2}^{\prime}}-\frac{1}{2}\left(\lambda_{4}+\lambda_{5}\right) v^{\prime 2}
$$

As before, the specific entries of $V_{\mathcal{N} s}^{\prime}$ are a consequence of the minimisation conditions, and the eigenvalues of the scalar mass matrices at an $\mathcal{N} s$ stationary point. As for the value of the potential, we have

$$
V_{\mathcal{N} s}=\frac{1}{2} A^{T} X_{\mathcal{N} s}=-\frac{1}{2} X_{\mathcal{N} s}^{T} B X_{\mathcal{N} s} .
$$

As mentioned earlier, another charge and $\mathrm{CP}$ conserving vacuum may arise in the model - one for which the singlet field acquires a VEV but the doublets do not. This type of vacuum — dubbed $S$ in [25] — would lead to massless electroweak gauge bosons and fermions, and as such it is unphysical. This stationary point exists if $m_{S}^{2}<0$, and the singlet VEV is found to be

$$
\left\langle\Phi_{S}\right\rangle^{2}=-\frac{2 m_{S}^{2}}{\lambda_{6}}
$$

The value of the potential at this stationary point is equal to

$$
V_{S}=-\frac{m_{S}^{4}}{2 \lambda_{6}}
$$

Both the $\mathcal{N}$ and $\mathcal{N} s$ phases can accommodate SM-like physics (provided that $v_{1}^{2}+v_{2}^{2} \sim$ $(246 \mathrm{GeV})^{2}$ and $v_{1}^{\prime 2}+v_{2}^{\prime 2} \sim(246 \mathrm{GeV})^{2}$, respectively), although each of these phases has a different phenomenology (for $\mathcal{N}$ dark matter candidates exist, for $\mathcal{N} s$ three $\mathrm{CP}$-even states mix with each other). We will now analyse the stability of both $\mathcal{N}$ and $\mathcal{N} s$ against the possible existence of deeper minima of different nature. For a large part of the parameter space of the model the minimisation conditions yield a single minimum, and its stability is ensured (at least at tree-level). However, for many combinations of the parameters of the potential, multiple minima can coexist. If the tunnelling time from a minimum of type $\mathcal{N}$ 
(or $\mathcal{N} s$ ) to a deeper minimum is smaller than the age of the universe then the corresponding set of parameters should be excluded. We note that we use a very conservative approach and only exclude those points where the survival probability is extremely small corresponding to tunnelling times much smaller than the age of the Universe. A detailed discussion is presented in section 3.2 .

\subsection{Stability of normal minima against charge breaking}

Since charge breaking minima have to be avoided, it is important to know under what circumstances a normal minimum is safe against eventual tunnelling to a deeper charge breaking minimum. In the $2 \mathrm{HDM}$ that question was answered $[8,9,14,15]$ in a conclusive manner: whenever a normal minimum exists, any charge breaking stationary point is necessarily a saddle point lying above the normal minimum. In the N2HDM, as we will now show, the situation is changed. Let us first define both of the possible charge breaking stationary points and introduce some notation concerning them.

- In the first charge breaking stationary point $\mathcal{C B}$ (denoted $I I b$ in [25]) the singlet field has no VEV, and the doublet VEVs are

$$
\left\langle\Phi_{1}\right\rangle_{\mathcal{C B}}=\frac{1}{\sqrt{2}}\left(\begin{array}{c}
0 \\
c_{1}
\end{array}\right), \quad\left\langle\Phi_{2}\right\rangle_{\mathcal{C B}}=\frac{1}{\sqrt{2}}\left(\begin{array}{c}
c_{2} \\
c_{3}
\end{array}\right), \quad\left\langle\Phi_{S}\right\rangle_{\mathcal{C B}}=0 .
$$

Consider also the vectors $X$ and $V^{\prime}$ evaluated at a $\mathcal{C B}$ stationary point, given by

$$
X_{\mathcal{C B}}=\langle X\rangle_{\mathcal{C B}}=\frac{1}{2}\left(\begin{array}{c}
c_{1}^{2} \\
c_{2}^{2}+c_{3}^{2} \\
c_{1} c_{3} \\
0 \\
0
\end{array}\right), \quad V_{\mathcal{C B}}^{\prime}=A+B X_{\mathcal{C B}}=\left(\begin{array}{c}
0 \\
0 \\
0 \\
0 \\
m_{S 1}^{2}
\end{array}\right)
$$

where $m_{S 1}^{2}=m_{S}^{2}+\lambda_{7} c_{1}^{2} / 2+\lambda_{8}\left(c_{2}^{2}+c_{3}^{2}\right) / 2$ is one of the squared scalar masses at the $\mathcal{C B}$ stationary point. The entries of $V_{\mathcal{C B}}^{\prime}$ are dictated by the $\mathcal{C B}$ minimisation conditions.

- In the second charge breaking stationary point $\mathcal{C B} s$ (denoted $s I I b$ in [25]) the singlet also acquires a VEV, the VEV configuration being given by

$$
\left\langle\Phi_{1}\right\rangle_{\mathcal{C B} s}=\frac{1}{\sqrt{2}}\left(\begin{array}{c}
0 \\
c_{1}^{\prime}
\end{array}\right), \quad\left\langle\Phi_{2}\right\rangle_{\mathcal{C B} s}=\frac{1}{\sqrt{2}}\left(\begin{array}{c}
c_{2}^{\prime} \\
c_{3}^{\prime}
\end{array}\right), \quad\left\langle\Phi_{S}\right\rangle_{\mathcal{C B} s}=c_{4}^{\prime} .
$$

Analogously to what we have done for the previous stationary points, we define the following vectors:

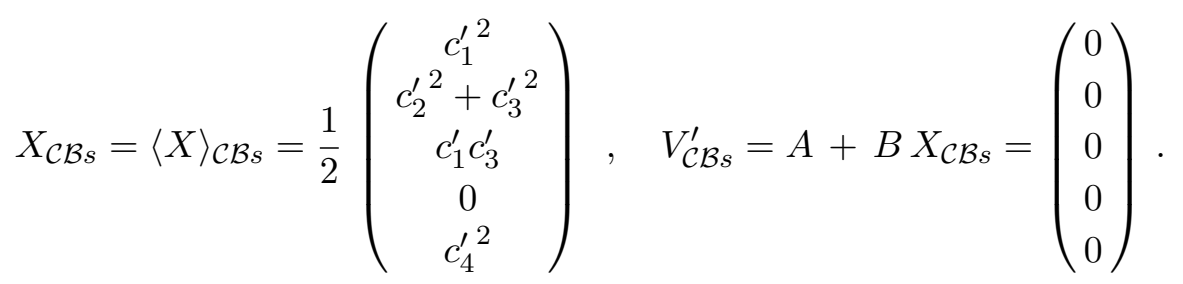

And again, the entries of $V_{\mathcal{C B} s}^{\prime}$ are dictated by the $\mathcal{C B} s$ minimisation conditions. 
The manipulation of the $X$ and $V^{\prime}$ vectors will allow us to establish analytical formulae relating the value of the potential at two coexisting stationary points. This technique was first used in ref. [14], and it essentially consists in following four basic steps: (1) perform the internal product of $X$ evaluated at one of the stationary points with $V^{\prime}$ evaluated at the second one; (2) repeat, with $X$ evaluated at the second stationary point and $V^{\prime}$ at the first one; (3) use the explicit formulas for $V^{\prime}$ to relate the previous internal products with the value of the potential at each stationary point; (4) the two internal products will have a common term, through which they can be related to one another, thus obtaining a relation between the potentials. The technique is best understood going through some explicit examples of its application, which we will now provide. Note that all of the following conclusions are derived at the tree-level and may be affected by higher order corrections.

We pause here to comment about higher order corrections. Corrections in the effective potential at NLO can be large in some regions of parameter space. With a proper choice of the renormalisation procedure, these large corrections can be moved into the quartic couplings of the potential. This would of course influence vacuum stability as well as the bounds from perturbative unitarity (for a discussion in the context of the CP-violating 2HDM (C2HDM), see for example [18]). However, such corrections change the relations between the input parameters and the observables, and are smoothed out in a scan covering the whole parameter space. We therefore do not expect the changes to be dramatic, as seen in [18].

Regarding the robustness of the results at higher orders, the Inert 2HDM was investigated in [16] using the one-loop effective potential. Differences to the tree-level results were observed but they were only found to be important if two minima were close to degenerate.

\subsubsection{Extrema $\mathcal{N}$ vs. $\mathcal{C B}$ and $\mathcal{C B} s$}

Let us assume that the parameters of the N2HDM are such that the potential has two stationary points, ${ }^{2}$ one of type $\mathcal{N}$ and another of type $\mathcal{C B}$. These extrema may or may not be minima, at this time we do not need to specify it. Let us then consider the vectors defined above, containing information about the VEVs and the minimisation conditions in each extremum, for $\mathcal{N}$ in eqs. (2.10), for $\mathcal{C B}$ in eqs. (2.20).

The internal product of the vectors $X_{\mathcal{C B}}$ and $V_{\mathcal{N}}^{\prime}$ yields

$$
X_{\mathcal{C B}}^{T} V_{\mathcal{N}}^{\prime}=\frac{m_{H^{ \pm}}^{2}}{2 v^{2}}\left[\left(v_{2} c_{1}-v_{1} c_{3}\right)^{2}+v_{1}^{2} c_{2}^{2}\right]
$$

which may also be written as

$$
X_{\mathcal{C B}}^{T} V_{\mathcal{N}}^{\prime}=X_{\mathcal{C B}}^{T}\left(A+B X_{\mathcal{N}}\right)=X_{\mathcal{C B}}^{T} A+X_{\mathcal{C B}}^{T} B X_{\mathcal{N}}
$$

From eq. (2.7), we know that the quantity $X_{\mathcal{C B}}^{T} A$ is twice the value of the potential at the extremum $\mathcal{C B}$,

$$
X_{\mathcal{C B}}^{T} A=2 V_{\mathcal{C B}}
$$

\footnotetext{
${ }^{2}$ That is, the minimisation equations of the N2HDM potential admit both solutions, for a given choice of parameters.
} 
and therefore, combining eqs. (2.23) and (2.24),

$$
X_{\mathcal{C B}}^{T} B X_{\mathcal{N}}=\frac{m_{H^{ \pm}}^{2}}{2 v^{2}}\left[\left(v_{2} c_{1}-v_{1} c_{3}\right)^{2}+v_{1}^{2} c_{2}^{2}\right]-2 V_{\mathcal{C B}}
$$

We now perform similar operations on the vectors $X_{\mathcal{N}}$ and $V_{\mathcal{C B}}^{\prime}$, yielding

$$
X_{\mathcal{N}}^{T} V_{\mathcal{C B}}^{\prime}=0 \Leftrightarrow X_{\mathcal{N}}^{T} A+X_{\mathcal{N}}^{T} B X_{\mathcal{C B}}=0 \text {. }
$$

The quantity $X_{\mathcal{N}}^{T} A$ is twice the value of the potential at the extremum $\mathcal{N}$, hence

$$
X_{\mathcal{N}}^{T} B X_{\mathcal{C B}}=-2 V_{\mathcal{N}}
$$

Since the matrix $B$ (defined in eq. (2.4)) is symmetric, the left-hand sides of eqs. (2.26) and (2.28) are identical. It is then trivial to obtain the following expression comparing the depth of the potential at both extrema,

$$
V_{\mathcal{C B}}-V_{\mathcal{N}}=\frac{m_{H^{ \pm}}^{2}}{4 v^{2}}\left[\left(v_{2} c_{1}-v_{1} c_{3}\right)^{2}+v_{1}^{2} c_{2}^{2}\right]
$$

Therefore, if $\mathcal{N}$ is a minimum one will have $m_{H^{ \pm}}^{2}>0$, and since the terms in square brackets above are surely positive, one will have $V_{\mathcal{C B}}-V_{\mathcal{N}}>0$. Thus we may conclude that:

If the potential has a minimum of type $\mathcal{N}$, any $\mathcal{C B}$ stationary point, if it exists, lies above $\mathcal{N}$.

As such, no tunnelling to a deeper $\mathcal{C B}$ minimum can occur.

Similar conclusions are reached when one compares $\mathcal{N}$ and $\mathcal{C B} s$ stationary points. Again, the starting point is to analyse the internal products of the vectors $X$ and $V^{\prime}$ for each stationary point. Using eqs. (2.22) and (2.10), we obtain

$$
\begin{aligned}
& X_{\mathcal{N}}^{T} V_{\mathcal{C B} s}^{\prime}=2 V_{\mathcal{N}}+X_{\mathcal{N}}^{T} B X_{\mathcal{C B} s}=0 \\
& X_{\mathcal{C B} s}^{T} V_{\mathcal{N}}^{\prime}=2 V_{\mathcal{C B} s}+X_{\mathcal{C B} s}^{T} B X_{\mathcal{N}}=\frac{1}{2}\left\{\frac{m_{H^{ \pm}}^{2}}{v^{2}}\left[\left(v_{2} c_{1}^{\prime}-v_{1} c_{3}^{\prime}\right)^{2}+v_{1}^{2} c_{2}^{\prime 2}\right]+m_{D}^{2} c_{4}^{2}\right\}
\end{aligned}
$$

and therefore, subtracting both equations one easily obtains

$$
V_{\mathcal{C B} s}-V_{\mathcal{N}}=\frac{1}{4}\left\{\frac{m_{H^{ \pm}}^{2}}{v^{2}}\left[\left(v_{2} c_{1}^{\prime}-v_{1} c_{3}^{\prime}\right)^{2}+v_{1}^{2} c_{2}^{2}\right]+m_{D}^{2}{c_{4}^{\prime}}^{2}\right\}
$$

If $\mathcal{N}$ is a minimum all of the squared scalar masses computed therein must be positive, and thus $V_{\mathcal{C B} s}-V_{\mathcal{N}}>0$.

If the potential has a minimum of type $\mathcal{N}$, any $\mathcal{C B}$ s stationary point, if it exists, lies above $\mathcal{N}$.

As such, no tunnelling to a deeper $\mathcal{C B}$ s minimum can occur. We can therefore conclude that minima of type $\mathcal{N}$ are completely stable against the possibility of charge breaking. 


\subsubsection{Extrema $\mathcal{N} s$ vs. $\mathcal{C B}$ and $\mathcal{C B} s$}

The analysis of the previous section can now be extended to the stability of $\mathcal{N} s$ minima - but as we will shortly see, the conclusions are different. Let us begin by comparing $\mathcal{N} s$ and $\mathcal{C B} s$ stationary points. As before, and using eqs. (2.14) and (2.22), we have

$$
\begin{aligned}
& X_{\mathcal{N} s}^{T} V_{\mathcal{C B} s}^{\prime}=2 V_{\mathcal{N} s}+X_{\mathcal{N} s}^{T} B X_{\mathcal{C B} s}=0 \\
& X_{\mathcal{C B} s}^{T} V_{\mathcal{N} s}^{\prime}=2 V_{\mathcal{C B} s}+X_{\mathcal{C B} s}^{T} B X_{\mathcal{N} s}=\left(\frac{m_{H^{ \pm}}^{2}}{2 v^{2}}\right)_{\mathcal{N} s}\left[\left(v_{2}^{\prime} c_{1}^{\prime}-v_{1}^{\prime} c_{3}^{\prime}\right)^{2}+v_{1}^{\prime 2} c_{2}^{\prime 2}\right],
\end{aligned}
$$

where we use the subscript "N $s$ " to emphasise that both the squared charged mass and the sum of the square of the VEVs concern the $\mathcal{N} s$ stationary point. From these equations, it is trivial to obtain

$$
V_{\mathcal{C B} s}-V_{\mathcal{N} s}=\left(\frac{m_{H^{ \pm}}^{2}}{4 v^{2}}\right)_{\mathcal{N} s}\left[\left(v_{2}^{\prime} c_{1}^{\prime}-v_{1}^{\prime} c_{3}^{\prime}\right)^{2}+v_{1}^{\prime 2}{c_{2}^{\prime}}^{2}\right] .
$$

Therefore, as before, if $\mathcal{N} s$ is a minimum, any $\mathcal{C B} s$ stationary point, if it exists, lies above it, and $\mathcal{N} s$ is stable against tunnelling to $\mathcal{C B} s$.

However, when one follows these steps whilst comparing $\mathcal{N} s$ and $\mathcal{C B}$ stationary points, one finds:

$$
\begin{aligned}
& X_{\mathcal{N} s}^{T} V_{\mathcal{C B}}^{\prime}=2 V_{\mathcal{N} s}+X_{\mathcal{N} s}^{T} B X_{\mathcal{C B}}=\frac{1}{2} s^{2} m_{S 1}^{2} \\
& X_{\mathcal{C B}}^{T} V_{\mathcal{N} s}^{\prime}=2 V_{\mathcal{C B}}+X_{\mathcal{C B}}^{T} B X_{\mathcal{N} s}=\left(\frac{m_{H^{ \pm}}^{2}}{2 v^{2}}\right)_{\mathcal{N} s}\left[\left(v_{2}^{\prime} c_{1}-v_{1}^{\prime} c_{3}\right)^{2}+v_{1}^{\prime 2} c_{2}^{2}\right],
\end{aligned}
$$

where, recall, $s$ is the singlet VEV at vacuum $\mathcal{N} s$ and $m_{S 1}^{2}$ one of the squared scalar masses at $\mathcal{C B}$. From this one obtains

$$
V_{\mathcal{C B}}-V_{\mathcal{N} s}=\left(\frac{m_{H^{ \pm}}^{2}}{4 v^{2}}\right)_{\mathcal{N} s}\left[\left(v_{2}^{\prime} c_{1}-v_{1}^{\prime} c_{3}\right)^{2}+v_{1}^{\prime 2} c_{2}^{2}\right]-\frac{1}{4} s^{2} m_{S 1}^{2} .
$$

There is now no mandatory relationship between the depths of these stationary points a priori, both of them can be minima, and none is privileged with respect to the other. As such - and numerical analyses prove this - there are situations in which a minimum $\mathcal{N} s$ coexists with a deeper $\mathcal{C B}$ minimum (or vice-versa). Thus we conclude:

Minima of type $\mathcal{N}$ s are stable against charge breaking for vacua of type $\mathcal{C B s ,}$ but not necessarily for those of type $\mathcal{C B}$.

The addition of a real singlet to the $2 \mathrm{HDM}$ qualitatively changes the vacuum stability behaviour of the scalar potential. Whereas in the $2 \mathrm{HDM}$ a normal minimum is guaranteed to be stable against any possible deeper charge breaking minimum, this is no longer the case in the N2HDM. The addition of the singlet field leads to possible instabilities, where a normal minimum which breaks the $\mathbb{Z}_{2}$ symmetry of the singlet might coexist with a charge breaking minimum (deeper or not) which does not break that same symmetry. However, any N2HDM normal minimum which preserves the $\mathbb{Z}_{2}$ symmetry of the singlet is perfectly stable against charge breaking. 


\subsection{Stability of normal minima against CP breaking}

Just like in the 2HDM, spontaneous CP breaking is possible in the N2HDM. In fact, CP in the N2HDM can be broken by two different minima, whereas in the 2HDM only one such vacuum can occur. Before discussing the stability of such vacua, however, some general considerations are in order: it only makes sense to study spontaneous CP breaking in models were $\mathrm{CP}$ is a well-defined symmetry, i.e. models that are invariant under a given $\mathrm{CP}$ symmetry, as is the case with the potential written in eq. (2.1). ${ }^{3}$ Also, care must be taken when discussing CP breaking, as it is not sufficient to have a complex valued VEV to be able to affirm that $\mathrm{CP}$ violation is occurring. In fact, there are situations for which $\mathrm{CP}$ may be preserved even if complex VEVs arise, and therefore one ought to look for other signs of $\mathrm{CP}$ violation, such as the couplings of scalar mass eigenstates to $Z$ bosons. In the N2HDM, however, with the field basis we chose, no such problems arise: if the vacuum state contains a complex VEV, CP breaking occurs and produces scalar states of indefinite $\mathrm{CP}$ properties. Finally, as was shown in ref. [25], in the N2HDM it is not possible to have coexisting $\mathrm{CP}$ breaking and charge breaking stationary points - if the minimisation conditions can be solved for one type (CP breaking or charge breaking) of vacua, then the other type (charge breaking or CP breaking) admits no solution. Thus the possibility of tunnelling between CP breaking and charge breaking minima is excluded a priori.

As before, the question under which conditions a given normal minimum is stable against tunnelling to a deeper $\mathrm{CP}$ breaking vacuum has been previously answered in the $2 \mathrm{HDM}[8,9,14,15]$, and the conclusion is analogous to the charge breaking case: whenever a normal minimum exists, any $\mathrm{CP}$ breaking stationary point is necessarily a saddle point lying above the normal minimum. In the N2HDM the situation of the $\mathrm{CP}$ breaking vacua will differ, as it did for the charge breaking case. The vacua where CP can be spontaneously broken are:

- The first CP-breaking stationary point $\mathcal{C P}$ (denoted IIa in [25]) preserves the $\mathbb{Z}_{2}$ symmetry of the singlet but one of the doublets has a complex VEV. We parametrise the VEVs as

$$
\left\langle\Phi_{1}\right\rangle_{\mathcal{C P}}=\frac{1}{\sqrt{2}}\left(\begin{array}{c}
0 \\
\bar{v}_{1}
\end{array}\right), \quad\left\langle\Phi_{2}\right\rangle_{\mathcal{C P}}=\frac{1}{\sqrt{2}}\left(\begin{array}{c}
0 \\
\bar{v}_{2}+\mathrm{i} \bar{v}_{3}
\end{array}\right), \quad\left\langle\Phi_{S}\right\rangle_{\mathcal{C P}}=0 .
$$

Let us define

$$
X_{\mathcal{C P}}=\langle X\rangle_{\mathcal{C P}}=\frac{1}{2}\left(\begin{array}{c}
\bar{v}_{1}^{2} \\
\bar{v}_{2}^{2}+\bar{v}_{3}^{2} \\
\bar{v}_{1} \bar{v}_{2} \\
\bar{v}_{1} \bar{v}_{3} \\
0
\end{array}\right), \quad V_{\mathcal{C P}}^{\prime}=A+\hat{B} X_{\mathcal{C P}}=\left(\begin{array}{c}
0 \\
0 \\
0 \\
0 \\
m_{\bar{D}}^{2}
\end{array}\right)
$$

\footnotetext{
${ }^{3} \mathrm{As}$ in the $2 \mathrm{HDM}$, more elaborate $\mathrm{CP}$ symmetries could be considered, but these would only impose extra restrictions on the parameters of the model.
} 
where $m_{\bar{D}}^{2}=m_{S}^{2}+\lambda_{7} \bar{v}_{1}^{2} / 2+\lambda_{8}\left(\bar{v}_{2}^{2}+\bar{v}_{3}^{2}\right) / 2$ is the squared mass of the singlet in this vacuum, and we introduce the matrix $\hat{B}$,

$$
\hat{B}=B+\left(\lambda_{4}-\lambda_{5}\right)\left(\begin{array}{ccccc}
0 & 1 & 0 & 0 & 0 \\
1 & 0 & 0 & 0 & 0 \\
0 & 0 & -2 & 0 & 0 \\
0 & 0 & 0 & 0 & 0 \\
0 & 0 & 0 & 0 & 0
\end{array}\right)
$$

The entries of $V_{\mathcal{C P}}^{\prime}$ are determined by the stationarity conditions and the form of the mass matrices at this vacuum.

- The second CP-breaking stationary point $\mathcal{C P} s$ (denoted sIIa in [25]) also breaks the $\mathbb{Z}_{2}$ symmetry of the singlet and gives a complex VEV to one of the doublets. The VEVs are therefore

$$
\left\langle\Phi_{1}\right\rangle_{\mathcal{C P} s}=\frac{1}{\sqrt{2}}\left(\begin{array}{c}
0 \\
\bar{v}_{1}^{\prime}
\end{array}\right), \quad\left\langle\Phi_{2}\right\rangle_{\mathcal{C P} s}=\frac{1}{\sqrt{2}}\left(\begin{array}{c}
0 \\
\bar{v}_{2}^{\prime}+\mathrm{i} \bar{v}_{3}^{\prime}
\end{array}\right), \quad\left\langle\Phi_{S}\right\rangle_{\mathcal{C P}}=\bar{v}_{4}^{\prime},
$$

and we define

$$
X_{\mathcal{C P} s}=\langle X\rangle_{\mathcal{C P} s}=\frac{1}{2}\left(\begin{array}{c}
\bar{v}_{1}^{\prime 2} \\
\bar{v}_{2}^{\prime 2}+\bar{v}_{3}^{\prime 2} \\
\bar{v}_{1}^{\prime} \bar{v}_{2}^{\prime} \\
\bar{v}_{1}^{\prime} \bar{v}_{3}^{\prime} \\
\bar{v}_{4}^{\prime 2}
\end{array}\right), \quad V_{\mathcal{C P} s}^{\prime}=A+\hat{B} X_{\mathcal{C P} s}=\left(\begin{array}{c}
0 \\
0 \\
0 \\
\left(\lambda_{4}-\lambda_{5}\right) \bar{v}_{1}^{\prime} \bar{v}_{3}^{\prime} \\
0
\end{array}\right)
$$

The entries of $V_{\mathcal{C} \mathcal{P} s}^{\prime}$ are determined by the stationarity conditions.

\subsubsection{Extrema $\mathcal{N}$ vs. $\mathcal{C P}$ and $\mathcal{C P} s$}

Following the strategy employed for comparing normal and charge breaking vacua, we now assume that the potential has two stationary points, one of type $\mathcal{N}$ and the other of type $\mathcal{C P}$, each of which may, or may not, be a minimum. With the vector definitions outlined above, we see that the internal product of the vectors $X_{\mathcal{C P}}$ and $V_{\mathcal{N}}^{\prime}$ yields

$$
X_{\mathcal{C P}}^{T} V_{\mathcal{N}}^{\prime}=\frac{m_{H^{ \pm}}^{2}}{2 v^{2}}\left[\left(v_{2} \bar{v}_{1}-v_{1} \bar{v}_{2}\right)^{2}+v_{1}^{2} \bar{v}_{3}^{2}\right]
$$

and thus

$$
X_{\mathcal{C P}}^{T} V_{\mathcal{N}}^{\prime}=X_{\mathcal{C P}}^{T}\left(A+B X_{\mathcal{N}}\right)=X_{\mathcal{C P}}^{T} A+X_{\mathcal{C P}}^{T} B X_{\mathcal{N}}
$$

Eq. (2.7) tell us that $X_{\mathcal{C P}}^{T} A=2 V_{\mathcal{C P}}$ and therefore

$$
X_{\mathcal{C P}}^{T} B X_{\mathcal{N}}=\frac{m_{H^{ \pm}}^{2}}{2 v^{2}}\left[\left(v_{2} \bar{v}_{1}-v_{1} \bar{v}_{2}\right)^{2}+v_{1}^{2} \bar{v}_{3}^{2}\right]-2 V_{\mathcal{C P}}
$$

With similar manipulations on $X_{\mathcal{N}}$ and $V_{\mathcal{C} P}^{\prime}$, we obtain

$$
X_{\mathcal{N}}^{T} V_{\mathcal{C P}}^{\prime}=0 \Leftrightarrow X_{\mathcal{N}}^{T} A+X_{\mathcal{N}}^{T} \hat{B} X_{\mathcal{C P}}=0 .
$$


Since $X_{\mathcal{N}}^{T} A=2 V_{\mathcal{N}}$, and with the definition of $\hat{B}$ in eq. (2.38), it is seen that

$$
X_{\mathcal{N}}^{T} \hat{B} X_{\mathcal{C P}}=-2 V_{\mathcal{N}} \Leftrightarrow X_{\mathcal{N}}^{T} B X_{\mathcal{C P}}=-\frac{1}{4}\left(\lambda_{4}-\lambda_{5}\right)\left[\left(v_{2} \bar{v}_{1}-v_{1} \bar{v}_{2}\right)^{2}+v_{1}^{2} \bar{v}_{3}^{2}\right]-2 V_{\mathcal{N}}
$$

Now, since the pseudoscalar squared mass for an $\mathcal{N}$ stationary point is given by

$$
m_{A}^{2}=m_{H^{ \pm}}^{2}+\frac{1}{2}\left(\lambda_{4}-\lambda_{5}\right) v^{2}
$$

we finally obtain

$$
V_{\mathcal{C P}}-V_{\mathcal{N}}=\frac{m_{A}^{2}}{4 v^{2}}\left[\left(v_{2} \bar{v}_{1}-v_{1} \bar{v}_{2}\right)^{2}+v_{1}^{2} \bar{v}_{3}^{2}\right]
$$

Thus, if $\mathcal{N}$ is a minimum one will have $m_{A}^{2}>0$, and therefore inevitably $V_{\mathcal{C P}}-V_{\mathcal{N}}>0$.

Following analogous steps for the $\mathcal{N}$ and $\mathcal{C} \mathcal{P} s$ stationary points, one arrives easily at the following formula comparing the depths of the potential at each stationary point,

$$
V_{\mathcal{C P} s}-V_{\mathcal{N}}=\frac{1}{4}\left\{\frac{m_{A}^{2}}{v^{2}}\left[\left(v_{2} \bar{v}_{1}^{\prime}-v_{1} \bar{v}_{2}^{\prime}\right)^{2}+v_{1}^{2} \bar{v}_{3}^{\prime 2}\right]+m_{D}^{2} \bar{v}_{4}^{\prime 2}\right\}
$$

Therefore, one reaches the same conclusions for $\mathcal{C P}$ and $\mathcal{C P} s$ stationary points, when they coexist with $\mathcal{N}$ :

If $\mathcal{N}$ is a minimum, it is deeper than any $\mathcal{C P}$ or $\mathcal{C P} s$ stationary points.

\subsubsection{Extrema $\mathcal{N} s$ vs. $\mathcal{C P}$ and $\mathcal{C P} s$}

The conclusions of the previous subsection do not extend unchanged to coexisting $\mathcal{N} s$ and $\mathcal{C P}$ or $\mathcal{C P} s$ stationary points. Starting with $\mathcal{C P} s$, we have

$$
X_{\mathcal{C P} s}^{T} V_{\mathcal{N} s}^{\prime}=\left(\frac{m_{H^{ \pm}}^{2}}{2 v^{2}}\right)_{\mathcal{N} s}\left[\left(v_{2}^{\prime} \bar{v}_{1}^{\prime}-v_{1}^{\prime} \bar{v}_{2}^{\prime}\right)^{2}+v_{1}^{\prime 2} \bar{v}_{3}^{\prime 2}\right]=2 V_{\mathcal{C P} s}+X_{\mathcal{C P} s}^{T} B X_{\mathcal{N} s}
$$

Also, we derive that

$$
X_{\mathcal{N} s}^{T} V_{\mathcal{C P} s}^{\prime}=2 V_{\mathcal{C P} s}+X_{\mathcal{N} s}^{T} \hat{B} X_{\mathcal{C P} s}
$$

and after similar calculations as before it may be seen that

$$
V_{\mathcal{C P} s}-V_{\mathcal{N} s}=\left(\frac{m_{A}^{2}}{4 v^{2}}\right)_{\mathcal{N} s}\left[\left(v_{2}^{\prime} \bar{v}_{1}^{\prime}-v_{1}^{\prime} \bar{v}_{2}^{\prime}\right)^{2}+v_{1}^{\prime 2} \bar{v}_{3}^{\prime 2}\right],
$$

and therefore if $\mathcal{N} s$ is a minimum it is certainly deeper than $\mathcal{C P} s$ - the same type of result we obtained when comparing $\mathcal{N}$ minima and $\mathrm{CP}$ ones. On the other hand, if we compare $\mathcal{N} s$ and $\mathcal{C P}$ stationary points, we obtain

$$
X_{\mathcal{C P}}^{T} V_{\mathcal{N} s}^{\prime}=\left(\frac{m_{H^{ \pm}}^{2}}{2 v^{2}}\right)_{\mathcal{N} s}\left[\left(v_{2}^{\prime} \bar{v}_{1}-v_{1}^{\prime} \bar{v}_{2}\right)^{2}+v_{1}^{\prime 2} \bar{v}_{3}^{2}\right]=2 V_{\mathcal{C P}}+X_{\mathcal{C P}}^{T} B X_{\mathcal{N} s} .
$$

Also, it is easy to obtain

$$
X_{\mathcal{N} s}^{T} V_{\mathcal{C P}}^{\prime}=\frac{1}{2} m_{\bar{D}}^{2} s^{2}=2 V_{\mathcal{N} s}+X_{\mathcal{N} s}^{T} \hat{B} X_{\mathcal{C P}}
$$


and hence, after trivial manipulations,

$$
V_{\mathcal{C P}}-V_{\mathcal{N} s}=\left(\frac{m_{A}^{2}}{4 v^{2}}\right)_{\mathcal{N} s}\left[\left(v_{2}^{\prime} \bar{v}_{1}-v_{1}^{\prime} \bar{v}_{2}\right)^{2}+v_{1}^{\prime 2} \bar{v}_{3}^{2}\right]-\frac{1}{4} m_{\bar{D}}^{2} s^{2} .
$$

This expression shows - as for the pair $\mathcal{N} s, \mathcal{C B}$ - that $\mathcal{N} s$ is not necessarily stable against tunnelling to a deeper $\mathcal{C P}$ minimum.

\section{Minima of type $\mathcal{N} s$ are stable against CP-breaking minima of type $\mathcal{C P} s$, but not against those of type $\mathcal{C P}$.}

\subsection{Other coexisting neutral minima}

Another possibility for vacuum instability is the existence of multiple minima of types $\mathcal{N}$, $\mathcal{N} s$ or even $S$. If for instance two $\mathcal{N}$ and $\mathcal{N} s$ stationary points coexist, we can follow similar steps to those outlined in the previous sections and arrive at the following formula relating the depths of the potential:

$$
V_{\mathcal{N} s}-V_{\mathcal{N}}=\frac{1}{4}\left[\left(\frac{m_{H^{ \pm}}^{2}}{4 v^{2}}\right)_{\mathcal{N}}-\left(\frac{m_{H^{ \pm}}^{2}}{4 v^{2}}\right)_{\mathcal{N} s}\right]\left(v_{1} v_{2}^{\prime}-v_{2} v_{1}^{\prime}\right)^{2}+\frac{1}{4} m_{D}^{2} s^{2} .
$$

Therefore, we see that since either one of $\mathcal{N}$ or $\mathcal{N} s$ can be minima, none of them is guaranteed to be deeper than the other. Therefore, though $\mathcal{N}$ is stable against tunnelling to a deeper charge breaking or CP breaking minimum, it is not guaranteed to be stable against a deeper $\mathcal{N} s$ vacuum. Likewise, an $\mathcal{N} s$ minimum, which is safe against tunnelling to possible charge breaking or $\mathrm{CP}$ breaking minima, may be unstable against a deeper $\mathcal{N}$ minimum. Nonetheless, we can derive another conclusion considering this formula in tandem with the results of previous sections:

If the parameters of the potential are such that $\mathcal{N}$ and $\mathcal{N} s$ minima coexist in the potential, then the global minimum of the potential preserves charge and $C P$.

The demonstration is simple: though from eq. (2.55) we cannot be certain whether $\mathcal{N}$ or $\mathcal{N} s$ is the global minimum, the existence of an $\mathcal{N}$ minimum places it certainly below any charge/CP breaking stationary points that might exist. Therefore, the conclusion becomes that either $\mathcal{N}$ or $\mathcal{N} s$ is the global minimum.

The other possibility still in play would be the coexistence of an $\mathcal{N}$ (or $\mathcal{N} s$ ) minimum with an $S$ minimum of the type described in eqs. (2.17) and (2.18), where only the singlet acquires a VEV. This is the simplest possibility of vacuum instability to verify: provided we find a solution of the $\mathcal{N}$ type, it will be safe against tunnelling to an $S$ minimum provided we verify the following three conditions:

- Since the $S$ vacuum only exists if $m_{S}^{2}<0$, we need not worry about tunnelling from $\mathcal{N}$ to $S$ if $m_{S}^{2}>0$.

- If however $m_{S}^{2}<0$, then the $\mathcal{N}$ vacuum is deeper than $S$ if $V_{\mathcal{N}}$ is smaller than $V_{S}$, with $V_{S}$ having a very simple form given by eq. (2.18). 
- If $m_{S}^{2}<0$ and $V_{\mathcal{N}}>V_{S}$ then the tunnelling time between both vacua must be computed.

Likewise for an $\mathcal{N} s$ vacuum, the analogous conditions for stability of $\mathcal{N} s$ would hold.

Finally, there is still another possibility for instability of vacua of types $\mathcal{N}$ (or $\mathcal{N} s$ ): that the minimisation conditions of the N2HDM may yield more than one solution for a given type of vacuum. This means that a solution of the type $\mathcal{N} \equiv\left\langle\left\{\Phi_{1}, \Phi_{2}, \Phi_{S}\right\}\right\rangle=$ $\left\{v_{1}, v_{2}, 0\right\} / \sqrt{2}$ exists, with $v_{1}^{2}+v_{2}^{2}=246^{2} \mathrm{GeV}^{2}$, as well as another, $\mathcal{N}^{\prime} \equiv\left\{w_{1}, w_{2}, 0\right\} / \sqrt{2}$ exists, with $w_{1}^{2}+w_{2}^{2} \neq 246^{2} \mathrm{GeV}^{2}$. This possibility already arises in the 2HDM [8-12] - therein dubbed "panic vacua" - and it remains in the N2HDM as an avenue for instability of the $\mathcal{N}$ vacuum (and also of the $\mathcal{N} s$ one, since the minimisation equations of the potential may well yield more than one solution of type $\mathcal{N} s$ ). We do not study this possibility analytically, but it is included in the numerical analysis presented in section 3 .

We end this section with a very interesting scenario for the limit $m_{12}^{2}=0$, when all symmetries are exact. The $\mathcal{N}$ and $\mathcal{N} s$ stationary points are related by eq. (2.55). This equation can re-written as

$$
V_{\mathcal{N} s}-V_{\mathcal{N}}=\frac{m_{12}^{2}}{16}\left[\frac{1}{v_{1} v_{2}}-\frac{1}{v_{1}^{\prime} v_{2}^{\prime}}\right]+\frac{1}{4} m_{D}^{2} s^{2} .
$$

It we set $m_{12}^{2}=0$, and $\mathcal{N}$ is a minimum it is a global minimum because not only $V_{\mathcal{N} s}-V_{\mathcal{N}}>$ 0 , but also because we proved before that it is stable with respect to other charge breaking or CP breaking minima. However, this conclusion is only valid provided both doublet VEVs are non-zero, that is, the only dark matter candidate has origin in the singlet.

\subsection{Vacuum stability}

The results of the previous sections show that, unlike what happened for the 2HDM, when normal minima occur in the N2HDM they are not necessarily the global minima of the model. We summarise the results we obtained in table 1 , where we illustrate the relation between the various types of possible minima. If a minimum of type $\mathcal{N}$ exists (i.e. a minimum where the singlet has no VEV and its discrete symmetry is preserved even after spontaneous symmetry breaking) then $\mathcal{N}$ is certainly deeper than any charge or CP breaking stationary points that the potential might have - the stability of $\mathcal{N}$ against $\mathrm{CP}$ or charge breaking is perfectly guaranteed in the model. In fact, it is even possible to demonstrate (see appendix A) that in this situation any charge breaking stationary points are necessarily saddle points: an $\mathcal{N}$ minimum implies that at least one, but not all, of the squared masses of a $\mathcal{C B}(s)$ stationary point is negative. Presumably the same applies to $\mathcal{C P}(s)$ stationary points as well, assuming the $2 \mathrm{HDM}$ analysis generalizes. Of course, for considerations of stability, the nature (minimum, maximum, saddle point) of extrema that lie above $\mathcal{N}$ is of no consequence.

The stability found for $\mathcal{N}$ minima does not hold, however, for minima of type $\mathcal{N} s$ : for these - the discrete symmetry of the singlet is spontaneously broken in addition to EW symmetry - coexistence with minima of certain types is indeed possible. An $\mathcal{N} s$ minimum will certainly be deeper than any stationary points of types $\mathcal{C B} s$ or $\mathcal{C P} s$ - which break, 


\begin{tabular}{|lccccccc|}
\hline Extrema & $\mathcal{N}$ & $\mathcal{N} s$ & $\mathcal{C B}$ & $\mathcal{C B} s$ & $\mathcal{C P}$ & $\mathcal{C P} s$ & $S$ \\
\hline $\mathcal{N}$ & $\times$ & $\times$ & Stability & Stability & Stability & Stability & $\times$ \\
$\mathcal{N} s$ & $\times$ & $\times$ & $\times$ & Stability & $\times$ & Stability & $\times$ \\
\hline
\end{tabular}

Table 1. Stability of extrema of types $\mathcal{N}$ and $\mathcal{N} s$ in the potential. For a given pair of extrema, "Stability" means that if one of them is a minimum, the other is necessarily above it. A pair of "Undefined" extrema (marked in the table with "x") means that both of them can be simultaneously minima, and neither is guaranteed to be the deepest one, depending on the choice of parameters.

respectively, charge conservation and $\mathrm{CP}$ symmetry, and also break the discrete symmetry of the singlet. But it is possible to have coexisting $\mathcal{N} s$ and $\mathcal{C B}$ or $\mathcal{C P}$ minima - which break, respectively, charge conservation and CP symmetry, but do not break the discrete symmetry of the singlet.

These results underline the curiously unique nature of the vacuum structure in the 2HDM, where the existence of a minimum of a given nature automatically implies that no minima of different types may exist. That property is not shared by models with a different scalar content - even in models with a simpler scalar content, such as the doublet + singlet (real or complex) model, the vacuum structure is much more complex, and no general, 2HDM-like conclusions may be drawn [37]. In models with more than two doublets the 2HDM stability also breaks down, at least concerning charge breaking [38]. What the analysis above has also shown is that the mere addition of just a real singlet to the $2 \mathrm{HDM}$ is enough to qualitatively change the vacuum structure of the model. The N2HDM preserves some of the nice vacuum properties of the $2 \mathrm{HDM}-$ wherein the $\mathcal{N}$ minimum mimics the stability behaviour of the normal minima of the $2 \mathrm{HDM}$ - but when $\mathcal{N} s$ minima are considered, the possibility of tunnelling to deeper minima of different types arises.

\section{$3 \quad$ Numerical analysis}

In order to illustrate the impact of the N2HDM vacuum structure on the phenomenologically relevant regions of the parameter space we perform a numerical study. We study combinations of parameters that are allowed by all available theoretical and experimental constraints and analyse their vacuum structure. We first outline our method for scanning the parameter space and present the constraints we apply. In order to judge whether deeper minima are indeed excluded it is necessary to calculate the tunnelling time from the EW vacuum. We use the method developed in [22] to numerically study the vacuum structure of these parameter points and estimate the lifetime of their EW vacua.

\subsection{Parameter scan}

We performed a scan of the N2HDM parameter space using an improved private version of ScannerS [25, 39-41]. We generated parameter points where the EW vacuum is of type $\mathcal{N} s$ since - following the analytical analysis - this is the most interesting case for vacuum stability. All of the resulting parameter points fulfil the applied theoretical constraints and are compatible with the applied current experimental constraints at the $2 \sigma$ level. 
The included theoretical constraints are tree-level perturbative unitarity [25] as well as boundedness from below [42]. A global minimum of the scalar potential only exists at finite field values if eq. (2.1) is bounded from below. This is a prerequisite for any study of vacuum stability. The allowed region is given by

$$
\Omega_{1} \cup \Omega_{2}
$$

with

$$
\Omega_{1}=\left\{\lambda_{1,2,6}>0 ; \sqrt{\lambda_{1} \lambda_{6}}+\lambda_{7}>0 ; \sqrt{\lambda_{2} \lambda_{6}}+\lambda_{8}>0 ; \sqrt{\lambda_{1} \lambda_{2}}+\lambda_{3}+D>0 ; \lambda_{7}+\sqrt{\frac{\lambda_{1}}{\lambda_{2}}} \lambda_{8} \geq 0\right\}
$$

and

$$
\begin{aligned}
\Omega_{2}= & \left\{\lambda_{1,2,6}>0 ; \lambda_{2} \lambda_{6} \geq \lambda_{8}^{2} ; \sqrt{\lambda_{1} \lambda_{6}}>-\lambda_{7} \geq \sqrt{\frac{\lambda_{1}}{\lambda_{2}}} \lambda_{8}\right. \\
& \left.\sqrt{\left(\lambda_{7}^{2}-\lambda_{1} \lambda_{6}\right)\left(\lambda_{8}^{2}-\lambda_{2} \lambda_{6}\right)}>\lambda_{7} \lambda_{8}-\left(D+\lambda_{3}\right) \lambda_{6}\right\}
\end{aligned}
$$

and depends on the discriminant

$$
D=\min \left(\lambda_{4}-\left|\lambda_{5}\right|, 0\right) .
$$

In contrast to earlier works [25, 43] we do not impose absolute stability of the EW vacuum as a theoretical constraint since we want to study the vacuum structure in detail and take into account that metastable regions of the parameter space are allowed.

The experimental constraints include bounds from flavour physics in the $m_{H^{ \pm}-\tan } \beta$ plane [44] — the $B_{d} \rightarrow \mu \mu$ constraint being the strongest in type I. We also require compatibility with the oblique parameters $S, T$ and $U[45,46]$ including the full correlation between these quantities [44]. We check for agreement with the collider Higgs data using HiggsBounds (v5.3.2beta) [47-51] and HiggsSignals (v2.2.3beta) [49, 52-54]. With HiggsBounds we check for $2 \sigma$ compatibility with all searches for additional scalars, and with HiggsSignals we employ a cut on $\Delta \chi^{2}=\chi_{\mathrm{N} 2 \mathrm{HDM}}^{2}-\chi_{\mathrm{SM}}^{2}<6.18$ (corresponding approximately to a $2 \sigma$ region). This cut ensures that the N2HDM predictions yield a $\chi^{2}$ in the fit to the LHC Higgs data that is at most $2 \sigma$ worse than the one of the SM. The required model predictions for branching ratios and total widths are obtained from N2HDECAY $[25,55]$ and the hadron collider production cross sections from SusHi $[56,57]$.

We use this setup to generate a sample of valid parameter points on which to study the vacuum structure and vacuum stability. One of the CP-even, neutral Higgs masses is fixed to

$$
m_{H_{x}}=m_{h_{125}}=125.09 \mathrm{GeV} .
$$

The remaining input parameters are independently drawn from uniform distributions with the ranges given in table 2. The three mixing angles in the CP-even scalar sector are scanned through their whole allowed range. In this work we only consider the N2HDM of 


\begin{tabular}{|cccccc|}
\hline & $m_{H_{y}}, m_{H_{z}}, m_{A}$ & $m_{H^{ \pm}}$ & $\tan \beta$ & $m_{12}^{2}$ & $v_{S}$ \\
\hline $\min$ & $30 \mathrm{GeV}$ & $150 \mathrm{GeV}$ & 0.8 & $0 \mathrm{GeV}^{2}$ & $1 \mathrm{GeV}$ \\
$\max$ & $1.5 \mathrm{TeV}$ & $1.5 \mathrm{TeV}$ & 20 & $5 \times 10^{5} \mathrm{GeV}^{2}$ & $3 \mathrm{TeV}$ \\
\hline
\end{tabular}

Table 2. Input parameter ranges for the N2HDM parameter scan $(y, z \in\{1,2,3\})$. The three mixing angles $\alpha_{1,2,3}$ in the CP-even scalar sector are scanned through their whole allowed range.

type I, i.e. where all fermions couple to $\Phi_{2}$, just mentioning briefly the results for a type II model as the vacuum structure and vacuum stability behaviour is unaffected by the choice of Yukawa type. Note that we do not specify a mass ordering for $m_{H_{x, y, z}}$ - the $h_{125}$ can be the lightest or heaviest state as well as the one in between.

\subsection{Numerical vacuum stability}

We use the approach presented in [22] to numerically study the vacuum structure and vacuum stability of the obtained parameter points. This approach is a highly efficient and numerically reliable method to study vacuum stability at the tree-level in BSM models with extended scalar sectors. We will now give a short review of our approach and refer to [22] for more details.

Our code uses polynomial homotopy continuation (PHC) (see e.g. [58] or [59]) to find all stationary points of the scalar potential eq. (2.1). This method reliably finds all solutions of a system of polynomial equations - in our case given by

$$
\frac{\partial V}{\partial \varphi_{i}}=0
$$

for the real component fields $\varphi_{i}$ of the doublets and singlet. The value of the scalar potential eq. (2.1) at each of these stationary points is then compared to the depth of the EW vacuum. If there is no stationary point deeper than the EW vacuum we consider the EW vacuum at this parameter point as absolutely stable. If stationary points deeper than the EW vacuum exist we calculate the tunnelling time to each of these deeper extrema. The decay width per (space-)volume $V_{S}$ to tunnel to a deeper point in field space is [60,61]

$$
\frac{\Gamma}{V_{S}}=K e^{-B}
$$

We approximate the tunnelling path by a straight path connecting the two minima in field space and use the semi-analytic solution given in [62] along this path to obtain the bounce action $B$. The prefactor $K$ is a subdominant contribution requiring an involved calculation and is therefore estimated on dimensional grounds. We consider the vacuum of the potential for a given parameter point to be short lived and the corresponding deeper minimum dangerous if

$$
B<390 \text {. }
$$

This is a conservative estimate where only vacua with a survival probability through the age of the universe

$$
P \ll 1-5 \sigma \sim 5.73 \times 10^{-7}
$$




\begin{tabular}{|lcccc|}
\hline & $\mathcal{N} s^{\prime}$ & $\mathcal{N}$ & $\mathcal{C B}$ & $\mathcal{C P}$ \\
\hline exists & $0.05 \%$ & $23.3 \%$ & $4.49 \%$ & $2.80 \%$ \\
deep & $0.0015 \%$ & $20.9 \%$ & $4.11 \%$ & $2.55 \%$ \\
dangerous & $0 \%$ & $6.89 \%$ & $1.12 \%$ & $0.678 \%$ \\
\hline
\end{tabular}

Table 3. Percentage of phenomenologically viable points that have a second minimum in addition to an EW vacuum of type $\mathcal{N} s$. In the first line we present the percentage of coexisting minima, in the second line the ones that are deeper and in the third line the dangerous, short-lived, ones. The minima of type $\mathcal{N} s^{\prime}$ have VEVs like those of $\mathcal{N} s$ but such that $v \neq 246 \mathrm{GeV}$, and differ from the EW vacuum in depth.

are considered short lived. We emphasise that the method developed in [22] is based on the straight path approximation as studied in [63]. It gives a good estimation for the bounce action with an accuracy of about $10 \%$. We have verified that our results remain qualitatively unchanged when varying the bound from eq. (3.8) by this margin.

\subsection{Discussion}

In this section we present a numerical and phenomenological analysis of the N2HDM vacuum structure and vacuum stability. The analysis is based on the sample of $10^{6}$ phenomenologically viable parameter points generated according to section 3.1. We aim to investigate whether the possible coexistence of minima discussed analytically in section 2

- is found in a substantial region of the N2HDM parameter space that is compatible with current theoretical and experimental constraints,

- can be directly related to phenomenological observations at colliders.

Since we assume the EW vacuum to be of type $\mathcal{N} s$ the potentially dangerous minima are $\mathcal{C B}, \mathcal{C P}, \mathcal{N}$, and a second different minimum of type $\mathcal{N} s$ (see below for a discussion of minima of type $S$ ). Unless otherwise stated, in the following we will distinguish three possibilities for these potentially dangerous vacua:

- they coexist with the EW vacuum (shown in green in the following plots),

- they are also deeper than the EW vacuum (shown in blue in the following plots),

- they are additionally dangerous, i.e. tunnelling from the EW vacuum is fast (as defined in eq. (3.8)) (shown in red in the following plots).

Table 3 shows the prevalence of these cases for the different possible secondary minima in our sample. While the precise numbers in table 3 have no physical significance as they depend on the applied method for sampling the parameter space, the displayed results clearly show that the possibilities discussed in section 2 remain relevant even after all other applicable constraints are considered. Especially, dangerous minima of type $\mathcal{N}$ (the dark matter phase with wrong EW symmetry breaking pattern) occur frequently in our sample. Table 3 also shows that the requirement of absolute stability would correspond to 

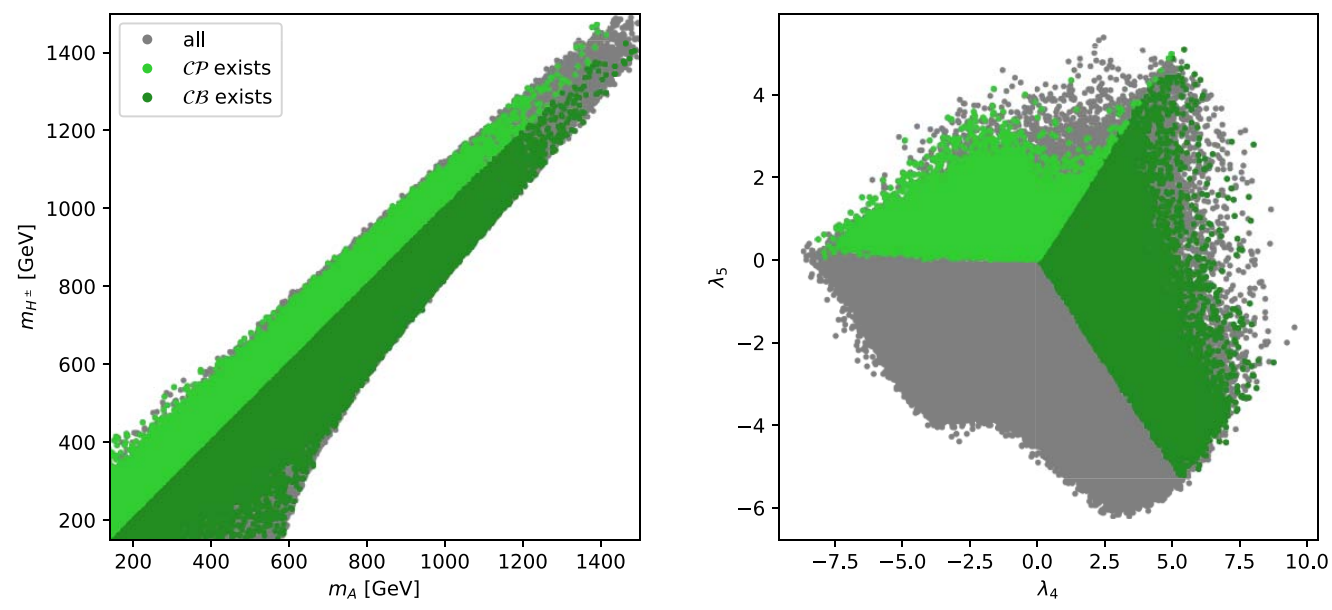

Figure 1. The distribution of secondary charge and CP breaking minima. The left plot shows the plane of the CP-odd Higgs mass $m_{A}$ and charged Higgs mass $m_{H^{ \pm}}$. The right plot shows the plane of the scalar potential parameters $\lambda_{4}$ and $\lambda_{5}$. In grey we show all parameter points fulfilling the theoretical and experimental constraints. On top we show the points where a secondary minimum of type $\mathcal{C B}$ (dark green) or $\mathcal{C P}$ (light green) exists.

a substantially stronger constraint on the parameter space compared to the requirement that the EW vacuum should be sufficiently long-lived. As a consequence, important parts of the parameter space that are actually viable would be discarded if the requirement of absolute stability was imposed.

The only case missing in table 3 that is allowed by the analytical analysis are secondary minima of type $S$. However, we have not found a single parameter point in our sample where a stationary point of type $S$ is a minimum. This could mean that minima of type $S$ cannot coexist with an $\mathcal{N} s$ vacuum, that all points where this is possible are ruled out by current constraints, or that these minima are exceedingly rare. Either way, since secondary minima of type $S$ do not occur in our sample they are of limited phenomenological interest, and we will not discuss them further here.

Figure 1, left, shows the distribution of charge and CP breaking secondary minima in the plane of the pseudoscalar Higgs mass $m_{A}$ and charged Higgs mass $m_{H^{ \pm}}$. The overall distribution of the phenomenologically viable parameter points is primarily driven by the EW precision measurements which force the neutral Higgs bosons to be relatively close in mass to the charged Higgs boson. Note that parameter points without any secondary minima as well as parameter points with secondary $\mathcal{N}$ minima exist throughout the allowed region. In contrast, secondary $\mathcal{C B}$ minima only exist as long as $m_{A}>m_{H^{ \pm}}$while $\mathcal{C P}$ minima only exist when $m_{H^{ \pm}}>m_{A}$.

The origin of this strict separation - making $m_{H^{ \pm}}=m_{A}$ the boundary between regions where only one of these types of minima exists - can be understood analytically. The pseudoscalar and charged masses in an $\mathcal{N}$ minimum are such that (see eq. (2.46))

$$
m_{A}^{2}-m_{H^{ \pm}}^{2}=\frac{1}{2}\left(\lambda_{4}-\lambda_{5}\right) v^{2} .
$$


Then, it is easy to show for $\mathcal{C B}$ and $\mathcal{C P}$ extrema, with VEVs given by eqs. (2.19) and eqs. (2.36), that the eigenvalues of the scalar mass matrix are given by

$$
m_{\mathcal{C B}}^{2}=\frac{1}{2}\left(\lambda_{4}-\lambda_{5}\right)\left(c_{1}^{2}+c_{2}^{2}+c_{3}^{2}\right), \quad m_{\mathcal{C P}}^{2}=-\frac{1}{2}\left(\lambda_{4}-\lambda_{5}\right)\left(\bar{v}_{1}^{2}+\bar{v}_{2}^{2}+\bar{v}_{3}^{2}\right) .
$$

Thus a $\mathcal{C B}$ minimum will imply $\lambda_{4}-\lambda_{5}>0$ and therefore, according to (3.10), $m_{A}>m_{H^{ \pm}}$. Similarly, a $\mathcal{C P}$ minimum requires $\lambda_{4}-\lambda_{5}<0$ which then implies $m_{A}<m_{H^{ \pm}}$. The same behaviour can be seen in figure 1 , right, showing the plane of $\lambda_{4}$ and $\lambda_{5}$. The $\mathcal{N}$ minima are again scattered throughout the allowed parameter space while the $\mathcal{C P}$ and $\mathcal{C B}$ minima can only occur in sharply defined regions. Therefore, $\lambda_{4}=\lambda_{5}$ would be the expected border between the regions where $\mathcal{C P}$ and $\mathcal{C B}$ can exist. However, figure 1, right, shows that there is an additional region

$$
\lambda_{5}<0 \wedge \lambda_{4}<-\lambda_{5}
$$

where neither $\mathcal{C P}$ nor $\mathcal{C B}$ minima can exist (see appendix A for an explanation).

In figure 2 we compare the analytical result for the relative depth of $\mathcal{N}$ and $\mathcal{N} s$ vacua to the numerical results. The relative depth of an $\mathcal{N} s$ and $\mathcal{N}$ vacuum, as given by eq. (2.55), is shown as a function of $\tan \beta$ at the $\mathcal{N} s \mathrm{EW}$ vacuum. The plot only includes parameter points where a secondary $\mathcal{N}$ minimum exists and shows its depth relative to the depth of the $\mathcal{N} s$ EW vacuum. As expected, in all parameter points where $V_{\mathcal{N} s}-V_{\mathcal{N}}>0$ the $\mathcal{N}$ minimum is classified as either deep (blue points) or dangerous (red points). The parameter points with dangerous $\mathcal{N}$ only begin to appear if $V_{\mathcal{N} s}-V_{\mathcal{N}} \gtrsim 10^{7}$, and their distribution shows some dependence on $\tan \beta$. For small $\tan \beta \lesssim 2$ the $\mathcal{N}$ vacuum is only unstable if the depth difference is $\gtrsim 10^{9}$ while for large $\tan \beta \gtrsim 12$ the majority of deep $\mathcal{N}$ vacua in our sample is dangerous. ${ }^{4}$

So far we have illustrated how the analytical results of section 2 are reflected in the phenomenologically viable parameter space. We will now discuss the vacuum stability constraints arising from these secondary vacua. In imposing vacuum stability constraints we distinguish the following cases:

- parameter points where the EW vacuum is the only vacuum,

- absolutely stable parameter points where secondary minima exist but are never deep,

- long-lived parameter points where secondary vacua are deep but never dangerous,

- short-lived parameter points that have dangerous secondary minima.

Figure 3 clearly demonstrates the phenomenological impact of vacuum stability constraints. It shows the signal strength of $h_{125}$ in the $\gamma \gamma$ channel defined as

$$
\mu_{\gamma \gamma}=\frac{\sigma\left(p p \rightarrow h_{125}\right) \mathrm{BR}\left(h_{125} \rightarrow \gamma \gamma\right)}{\sigma\left(p p \rightarrow h_{\mathrm{SM}}\right) \operatorname{BR}\left(h_{\mathrm{SM}} \rightarrow \gamma \gamma\right)}
$$

\footnotetext{
${ }^{4}$ This is more clearly visible when reversing the plotting order of figure 2 and plotting the parameter points with deep but not dangerous $\mathcal{N}$ vacua on top.
} 


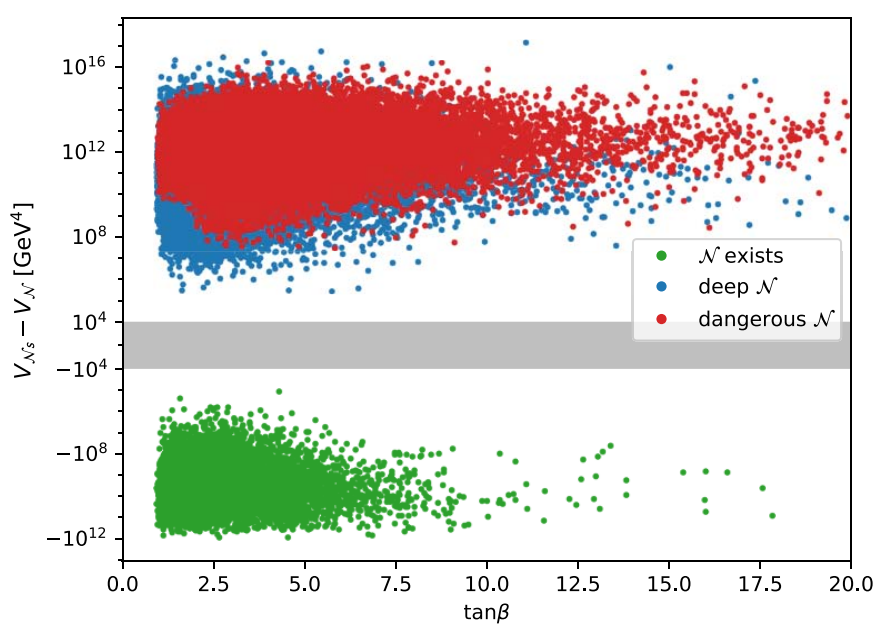

Figure 2. The difference in the value of the scalar potential between the EW $\mathcal{N} s$ vacuum and a secondary $\mathcal{N}$ minimum according to eq. (2.55) as a function of $\tan \beta$ at the $\mathrm{EW}$ vacuum. Only parameter points where a secondary $\mathcal{N}$ vacuum exists are shown. The color code is based on the results of the numerical analysis. The green parameter points have a secondary $\mathcal{N}$ minimum but tunnelling from the EW vacuum is not possible. For the blue parameter points tunnelling is possible but slow (see eq. (3.8)) while the EW vacuum in the red points (plotted on top) is short-lived for tunnelling to the $\mathcal{N}$ minimum.

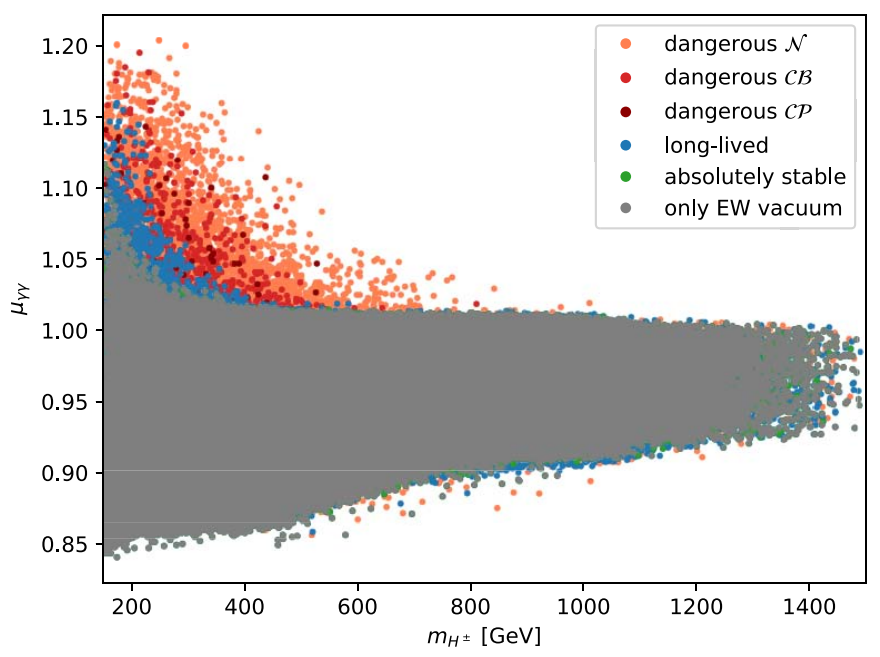

Figure 3. The signal strength $\mu_{\gamma \gamma}$ of $h_{125} \rightarrow \gamma \gamma$ as a function of the charged Higgs mass. The parameter points without any secondary minima (grey) are plotted on top, followed by the absolutely stable (green), and long-lived (blue) parameter points. Below these, the points with dangerous secondary minima are shown in different shades of red denoting the type of dangerous minimum present $(\mathcal{N}$ - light red, $\mathcal{C B}$ - red, $\mathcal{C P}$ - dark red $)$.

as a function of the charged Higgs mass. The short-lived (different shades of red) parameter points are plotted below the grey points, for which no secondary minima exist. This means that any region where only the red parameter points are visible is excluded by vacuum stability. One can see that significant parts of the parameter space corresponding to an 
enhanced signal strength, $\mu_{\gamma \gamma}>1$, are excluded because they have a dangerous $\mathcal{N}, \mathcal{C P}$ or $\mathcal{C B}$ minimum below the EW vacuum. If for instance a charged Higgs is found with a mass of $500 \mathrm{GeV}$, a bound of about $\mu_{\gamma \gamma} \lesssim 1.03$ in the N2HDM of type I can be derived from figure 3. If on the other hand the charged Higgs mass could be constrained to be larger than $250 \mathrm{GeV}$ (e.g. by a $500 \mathrm{GeV} e^{+} e^{-}$-collider) enhancements of $\mu_{\gamma \gamma}$ above 1.1 would be excluded in the N2HDM of type I by the vacuum stability constraint. One can also see from figure 3 that if the constraint of an absolutely stable EW vacuum were imposed, the blue points in figure 3, which indicate a long-lived EW vacuum, would be excluded, implying possibly misleading conclusions.

The reason for the behaviour observed in figure 3, i.e. the impact of vacuum stability on the allowed $\mu_{\gamma \gamma}$ values, is the $h_{125}$ coupling to a pair of charged Higgs bosons (defined in the appendix of [25]) as shown in figure 4 . This figure displays the impact of vacuum stability on the allowed values of the $h_{125} H^{+} H^{-}$coupling. Large negative values of this coupling are excluded by dangerous vacua. Negative values, however, lead to an enhancement of $\mu_{\gamma \gamma}$ through constructive interference with the $W^{ \pm}$loop. Note, that we have checked that there are no relevant effects from vacuum stability on the $h_{125}$ couplings to gauge bosons and to fermions. Therefore, $\mu_{\gamma \gamma}$ is the observable where the vacuum stability constraint is expected to have the largest impact since, among the currently measured observables, it has the highest sensitivity to the possible effects of a triple scalar coupling. The large impact of the vacuum stability constraint on $\mu_{\gamma \gamma}$ is specific to the N2HDM of type I. This is due to the fact that in type I all Yukawa couplings are rescaled by the same factor $c\left(h_{125} f \bar{f}\right)$. The cancellation of this factor which occurs in $\mu_{\gamma \gamma}$ in the approximation $\Gamma_{\text {tot }}\left(h_{125}\right) \approx \Gamma\left(h_{125} \rightarrow b \bar{b}\right)$,

$$
\begin{aligned}
\mu_{\gamma \gamma} & \approx c^{2}\left(h_{125} t \bar{t}\right) \frac{\Gamma\left(h_{125} \rightarrow \gamma \gamma\right)}{\Gamma\left(h_{125} \rightarrow b \bar{b}\right)} \frac{\Gamma_{\mathrm{tot}}\left(h_{\mathrm{SM}}\right)}{\Gamma\left(h_{\mathrm{SM}} \rightarrow \gamma \gamma\right)} \\
& \approx c^{2}\left(h_{125} t \bar{t}\right) \frac{\Gamma\left(h_{125} \rightarrow \gamma \gamma\right)}{c^{2}\left(h_{125} b \bar{b}\right) \Gamma\left(h_{\mathrm{SM}} \rightarrow \gamma \gamma\right)} \\
& =\frac{\Gamma\left(h_{125} \rightarrow \gamma \gamma\right)}{\Gamma\left(h_{\mathrm{SM}} \rightarrow \gamma \gamma\right)}
\end{aligned}
$$

leads to an increased sensitivity to $\Gamma\left(h_{125} \rightarrow \gamma \gamma\right)$ and thus to $g_{h_{125} H^{+} H^{-}}$. In contrast, for Yukawa types where $c\left(h_{125} t \bar{t}\right) \neq c\left(h_{125} b \bar{b}\right)$ (e.g. type II) the effect of vacuum stability constraints on $\mu_{\gamma \gamma}$ is no longer visible as the ratio of Yukawa couplings has a much stronger impact on the signal rate than the charged Higgs contribution to $\Gamma\left(h_{125} \rightarrow \gamma \gamma\right)$.

It is interesting to note that although the allowed range for $\mu_{\gamma \gamma}$ is very similar in the type I $2 \mathrm{HDM}$ [25] and in the type I N2HDM, a measurement of $\mu_{\gamma \gamma}$ above 1 for certain charged Higgs masses could exclude the N2HDM but be compatible with the 2HDM due to the different vacuum stability constraints.

Figure 5 shows vacuum stability constraints in the plane of the mass $m_{H_{2}}$ of the second lightest Higgs boson $H_{2}$, with a mass above $H_{1}=h_{125}$, and the signal strength $\mu_{\tau \tau}$ of $h_{125}$ (defined analogously to eq. (3.13)). In this case, there are hardly any regions where points can be clearly excluded due to the existence of a secondary dangerous vacuum. There are regions where only points with a non-stable vacuum exist, which can be either dangerous 


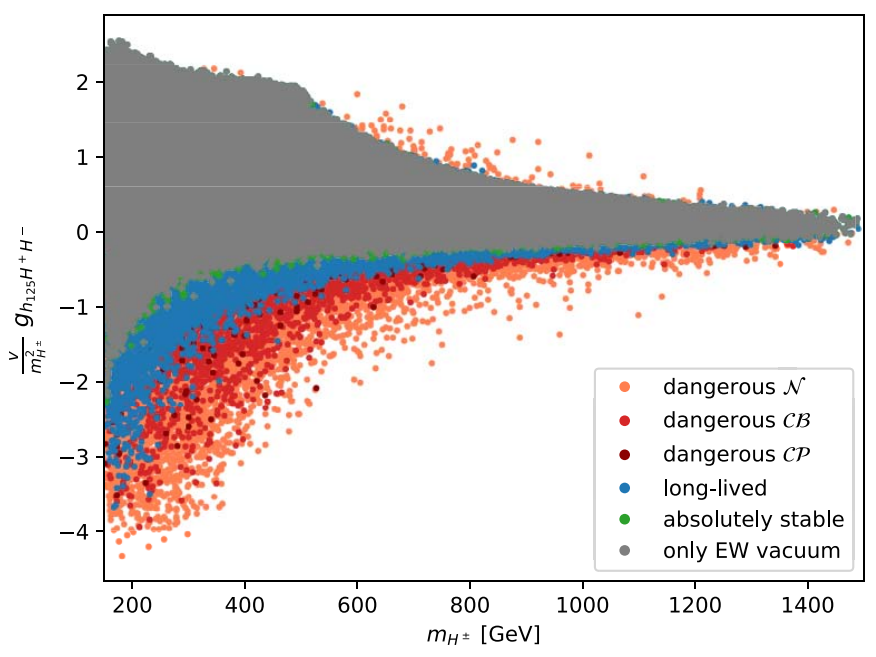

Figure 4. The normalised coupling $g_{h_{125} H^{+} H^{-}}$as a function of the charged Higgs mass. Colour code as in figure 3 .

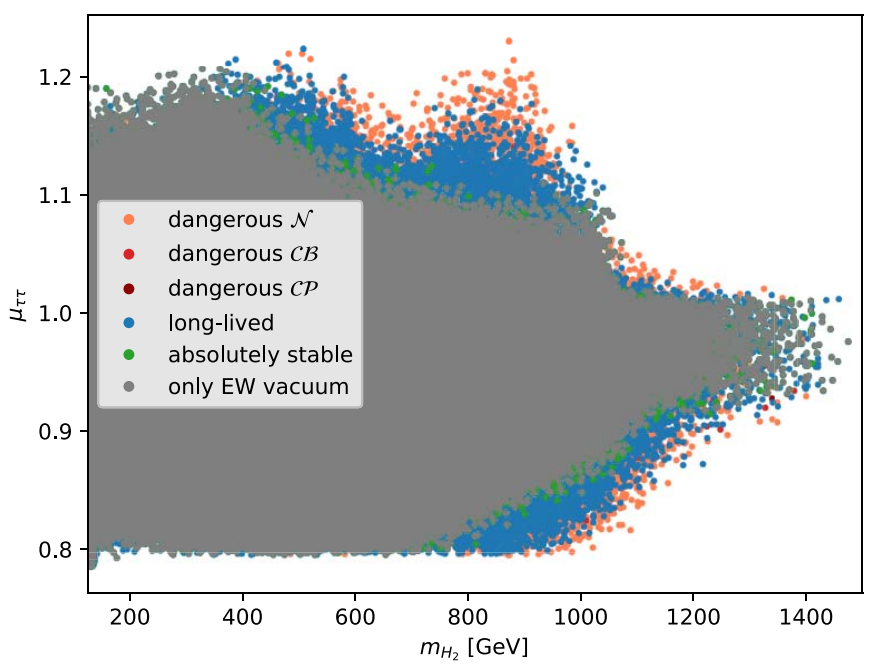

Figure 5. The signal strength $\mu_{\tau \tau}$ of $h_{125} \rightarrow \tau \tau$ as a function of the second lightest neutral scalar mass $m_{H_{2}}$. The parameter points without any secondary minima (grey) are plotted on top, followed by the absolutely stable (green), and long-lived (blue) parameter points. Below these, the points with dangerous secondary minima are shown in different shades of red denoting the type of dangerous minimum present $(\mathcal{N}$ - light red, $\mathcal{C B}$ - red, $\mathcal{C P}$ - dark red $)$.

or long-lived, but no direct bounds can be derived from the experimental measurements of $\mu_{\tau \tau}$. This is due to the fact that in contrast to figure 3 these regions are always populated by long-lived metastable vacua, so that allowed parameter points exist in these regions. Therefore, figure 5 clearly shows the phenomenological difference between requiring an absolutely stable EW vacuum (keeping only the grey and green parameter points) and a long-lived EW vacuum (additionally keeping the blue parameter points). As discussed above, enforcing absolute stability could lead to misleading phenomenological conclusions. 


\section{Conclusions}

We have performed a detailed analysis of the vacuum structure of the N2HDM, an extension of the SM by an extra doublet and an extra real singlet. We have shown that it is possible to derive analytical expressions to compare minima of different nature. In the case where the singlet has no VEV the conclusions are the same as for the 2HDM [14], that is, minima of different nature, $\mathcal{N}, \mathcal{C B}$ and $\mathcal{C P}$, never coexist. We have also shown analytically that when the singlet acquires a VEV, if a normal $\mathcal{N} s$ minimum exists, it is stable against tunnelling to a corresponding charge breaking $\mathcal{C B} s$ or CP-breaking $\mathcal{C P} s$ extremum. However, that conclusion no longer holds when comparing minima with and without singlet VEV. In fact, minima of different natures can coexist and potentially tunnel into each other. Moreover, it is known that in the $2 \mathrm{HDM}$ minima of type $\mathcal{N}$ are not unique [8-12] and the existence of a second, normal minimum (panic vacuum) can exist below the one with the correct EW symmetry breaking. In the N2HDM panic vacua of types $\mathcal{N}$ and $\mathcal{N} s$ can appear for EW vacua of either type. Additionally, minima of type $S$ with only a singlet VEV could also appear as panic vacua. However, we have not found a single parameter point in our sample where a stationary point of type $\mathrm{S}$ is a minimum.

Based on this analytical analysis we have conducted a numerical study to investigate the impact of the intricate N2HDM vacuum structure on the phenomenology of the model. We have generated a large sample of parameter points with an EW vacuum of type $\mathcal{N} s$ that fulfil all applicable theoretical and experimental constraints (without enforcing that the EW vacuum be a global minimum). This way, we were able to compare minima of different nature and identify regions of parameter space where the EW vacuum is the global minimum, where deeper minima exist but tunnelling is so slow that the EW vacuum is long-lived, and regions that are excluded because the tunnelling time is short compared to the age of the universe.

The first important conclusion of our study was that panic vacua of type $\mathcal{N}$, as well as charge breaking $\mathcal{C B}$, and $\mathrm{CP}$ breaking $\mathcal{C P}$ minima deeper than the $\mathrm{EW}$ vacuum appear in a significant portion of the (otherwise) phenomenologically viable parameter space. We have also shown the distribution of secondary $\mathcal{C B}$ and $\mathcal{C P}$ minima and established the boundaries of the disjunct parameter regions where these minima can exist.

Studying the impact of vacuum stability on collider observables we have found that a precise measurement of $\mu_{\gamma \gamma}$ above 1 could exclude the model on the grounds of vacuum stability alone, unless the charged Higgs is very light. This is due to the sensitivity of $\mu_{\gamma \gamma}$ to the triple Higgs coupling $g_{h_{125} H^{+} H^{-}}$, which is constrained by vacuum stability. If the Yukawa sector is of type I this effect is clearly visible in $\mu_{\gamma \gamma}$ because of an approximate cancellation between the modifications of the Yukawa couplings. In the study of other collider observables, such as $\mu_{\tau \tau}$, we showed that there are large regions where minima which are absolutely stable do not occur, but a long-lived EW vacuum exists. This illustrates the importance of including parameter regions with a metastable vacuum in phenomenological analyses, as enforcing absolute stability may lead to incorrect conclusions. 


\section{Acknowledgments}

$\mathrm{PF}$ and RS are supported in part by a CERN grant CERN/FIS-PAR/0002/2017, an FCT grant PTDC/FIS-PAR/31000/2017, by the CFTC-UL strategic project UID/FIS/00618/2019 and by the NSC, Poland, HARMONIA UMO2015/18/M/ST2/00518. MM acknowledges the contribution of the research training group GRK1694 'Elementary particle physics at highest energy and precision' and support by the Deutsche Forschungsgemeinschaft (DFG, German Research Foundation) under grant 396021762 - TRR 257. JW and GW acknowledge funding by the Deutsche Forschungsgemeinschaft under Germany's Excellence Strategy - EXC 2121 "Quantum Universe" - 390833306. JW acknowledges funding from the PIER Helmholtz Graduate School.

\section{A On the nature of stationary points}

We have shown that an $\mathcal{N}$ minimum is stable against tunnelling to deeper $\mathcal{C B}(s)$ or $\mathcal{C P}(s)$ extrema - if they exist, the $\mathcal{N}$ minimum is certainly deeper and no tunnelling to these extrema may occur. It is also possible to show that, if $\mathcal{N}$ is a minimum, any $\mathcal{C B}(s)$ stationary points that may exist are not only necessarily above it but cannot be minima themselves. Rather, they are saddle points.

We will demonstrate this nice property for the $\mathcal{C B} s$ case - the demonstration for the $\mathcal{C B}$ and $\mathcal{C P}(s)$ cases is similar. First recall that for a $\mathcal{C B} s$ extremum the vector $V^{\prime}$ is given

in eq. (2.22), and since $V_{\mathcal{C B} s}^{\prime}=A+B X_{\mathcal{C B} s}=0$, we will have $X_{\mathcal{C B} s}=-B^{-1} A$. Recalling the definition of $V_{\mathcal{N}}^{\prime}$, we may also write $A=V_{\mathcal{N}}^{\prime}-B X_{\mathcal{N}}$, and as such an alternate form for eq. (2.30) is

$$
X_{\mathcal{C} B s}^{T} V_{\mathcal{N}}^{\prime}=-V_{\mathcal{N}}^{\prime T}\left(B^{-1} A\right)=-V_{\mathcal{N}}^{\prime T} B^{-1}\left(V_{\mathcal{N}}^{\prime}-B X_{\mathcal{N}}\right)
$$

Since $V^{\prime T}{ }_{\mathcal{N}}^{T} X_{\mathcal{N}}=0$ (see eq. (2.10)), we find a different expression for (2.31), i.e.

$$
V_{\mathcal{C B} s}-V_{\mathcal{N}}=-\frac{1}{2} V_{\mathcal{N}^{\prime}}^{T} B^{-1} V_{\mathcal{N}}^{\prime}
$$

Now, we have shown that if $\mathcal{N}$ is a minimum then the right-hand-side of this matrix is positive (see eq. (2.31)). This therefore implies that in that situation the matrix $B^{-1}-$ and by extension the matrix $B-$ cannot be positive-definite. Therefore the matrix $B$ has at least one negative eigenvalue, but it certainly has positive ones - notice that the diagonal elements $B_{11}, B_{22}$ and $B_{66}$ are certainly positive so that the N2HDM potential is bounded from below, so $B$ necessarily has positive eigenvalues.

Let us now look at the squared scalar mass matrix, given by the second derivatives of the potential with respect to the real components of the doublets and singlet, $\varphi_{i}, i=1 \ldots 9$. We may write it as

$$
\begin{aligned}
{\left[M^{2}\right]_{i j} } & =\frac{\partial^{2} V}{\partial \varphi_{i} \partial \varphi_{j}}=\frac{\partial V}{\partial x_{l}} \frac{\partial^{2} x_{l}}{\partial \varphi_{i} \partial \varphi_{j}}+\frac{\partial^{2} V}{\partial x_{l} \partial x_{m}} \frac{\partial x_{l}}{\partial \varphi_{i}} \frac{\partial x_{m}}{\partial \varphi_{j}} \\
& =V_{l}^{\prime} \frac{\partial^{2} x_{l}}{\partial \varphi_{i} \partial \varphi_{j}}+B_{l m} \frac{\partial x_{l}}{\partial \varphi_{i}} \frac{\partial x_{m}}{\partial \varphi_{j}}
\end{aligned}
$$


where we introduced the matrix $B$ and the vector $V^{\prime}$ which are defined, respectively, in eqs. (2.4) and (2.6). Then, since for a $\mathcal{C B} s$ stationary point $V_{l}^{\prime}=0$, the mass matrix $\left[M^{2}\right]$ is reduced to the second term in the equation above. It is then rather easy to reproduce the calculation in section 5.2 of ref. [14] and deduce that one may simplify the expression of $\left[M^{2}\right]$ and obtain

$$
\left[M^{2}\right]=\left[\begin{array}{cc}
0 & 0 \\
0 & C^{T} B C
\end{array}\right],
$$

where $C$ is a $5 \times 5$ matrix depending only on the VEVs. Eq. (A.4) demonstrates that the eigenvalues of $\left[M^{2}\right]$ at a $\mathcal{C B} s$ stationary point will be all positive if and only if the matrix $B$ is positive definite. However, we have shown above that when $\mathcal{N}$ is a minimum, the matrix $B$ has at least one negative eigenvalue - and therefore $\left[M^{2}\right]$ has also at least one negative eigenvalue. However, since $B$ also has positive eigenvalues, so will $\left[M^{2}\right]$. Therefore, if $\mathcal{N}$ is a minimum then any $\mathcal{C B} s$ stationary point, it if exists, lies above $\mathcal{N}$ and is a saddle point, q.e.d.

We can now also justify the conditions of eq. (3.12) for the non-existence of neither $\mathcal{C B}$ or $\mathcal{C P}$ minima. The matrix $B$ determines the nature of the $\mathcal{C B} s$ stationary point, and one can also show that it does the same for the $\mathcal{C B}$ extrema. Checking now eqs. (2.4), we see that the $(3,3)$ entry of $B$ is $\lambda_{4}+\lambda_{5}$, and therefore, if $\lambda_{4}<-\lambda_{5}$ one of the diagonal elements of $B$ will be negative - thus $B$ cannot be positive definite, and consequently no $\mathcal{C B}$ minima can occur (only saddle points). This justifies the second condition of eq. (3.12). As for the first one $-\lambda_{5}<0$ - the nature of $\mathcal{C P}$ stationary points will, in analogy with the $\mathcal{C B}$ cases (and the $2 \mathrm{HDM}$, see [14]) be determined by a matrix of the quartic couplings. For the $\mathcal{C P}$ extrema, however, that matrix is not $B$ but rather the matrix $\hat{B}$, eq. (2.38). Observe then that the $(3,3)$ element of $\hat{B}$ is $2 \lambda_{5}$ - and therefore, if $\lambda_{5}<0$ no $\mathcal{C P}$ minima can occur since $\hat{B}$ cannot be positive definite.

Open Access. This article is distributed under the terms of the Creative Commons Attribution License (CC-BY 4.0), which permits any use, distribution and reproduction in any medium, provided the original author(s) and source are credited.

\section{References}

[1] P.W. Higgs, Broken symmetries, massless particles and gauge fields, Phys. Lett. 12 (1964) 132 [INSPIRE].

[2] P.W. Higgs, Broken Symmetries and the Masses of Gauge Bosons, Phys. Rev. Lett. 13 (1964) 508 [INSPIRE].

[3] P.W. Higgs, Spontaneous Symmetry Breakdown without Massless Bosons, Phys. Rev. 145 (1966) 1156 [INSPIRE].

[4] F. Englert and R. Brout, Broken Symmetry and the Mass of Gauge Vector Mesons, Phys. Rev. Lett. 13 (1964) 321 [INSPIRE].

[5] G.S. Guralnik, C.R. Hagen and T.W.B. Kibble, Global Conservation Laws and Massless Particles, Phys. Rev. Lett. 13 (1964) 585 [InSPIRE]. 
[6] G. Degrassi et al., Higgs mass and vacuum stability in the Standard Model at NNLO, JHEP 08 (2012) 098 [arXiv: 1205.6497] [INSPIRE].

[7] A.V. Bednyakov, B.A. Kniehl, A.F. Pikelner and O.L. Veretin, Stability of the Electroweak Vacuum: Gauge Independence and Advanced Precision, Phys. Rev. Lett. 115 (2015) 201802 [arXiv: 1507.08833] [INSPIRE].

[8] I.P. Ivanov, Minkowski space structure of the Higgs potential in 2HDM, Phys. Rev. D 75 (2007) 035001 [Erratum ibid. D 76 (2007) 039902] [hep-ph/0609018] [INSPIRE].

[9] I.P. Ivanov, Minkowski space structure of the Higgs potential in 2HDM. II. Minima, symmetries and topology, Phys. Rev. D 77 (2008) 015017 [arXiv:0710.3490] [INSPIRE].

[10] A. Barroso, P.M. Ferreira and R. Santos, Neutral minima in two-Higgs doublet models, Phys. Lett. B 652 (2007) 181 [hep-ph/0702098] [INSPIRE].

[11] A. Barroso, P.M. Ferreira, I.P. Ivanov, R. Santos and J.P. Silva, Evading death by vacuum, Eur. Phys. J. C 73 (2013) 2537 [arXiv:1211.6119] [inSPIRE].

[12] A. Barroso, P.M. Ferreira, I.P. Ivanov and R. Santos, Metastability bounds on the two Higgs doublet model, JHEP 06 (2013) 045 [arXiv:1303.5098] [INSPIRE].

[13] V. Branchina, F. Contino and P.M. Ferreira, Electroweak vacuum lifetime in two Higgs doublet models, JHEP 11 (2018) 107 [arXiv:1807.10802] [INSPIRE].

[14] P.M. Ferreira, R. Santos and A. Barroso, Stability of the tree-level vacuum in two Higgs doublet models against charge or CP spontaneous violation, Phys. Lett. B 603 (2004) 219 [Erratum ibid. B 629 (2005) 114] [hep-ph/0406231] [INSPIRE].

[15] A. Barroso, P.M. Ferreira and R. Santos, Charge and CP symmetry breaking in two Higgs doublet models, Phys. Lett. B 632 (2006) 684 [hep-ph/0507224] [INSPIRE].

[16] P.M. Ferreira and B. Swiezewska, One-loop contributions to neutral minima in the inert doublet model, JHEP 04 (2016) 099 [arXiv: 1511. 02879] [INSPIRE].

[17] P. Basler, M. Krause, M. Muhlleitner, J. Wittbrodt and A. Wlotzka, Strong First Order Electroweak Phase Transition in the CP-Conserving 2HDM Revisited, JHEP 02 (2017) 121 [arXiv: 1612.04086] [INSPIRE].

[18] P. Basler, M. Mühlleitner and J. Wittbrodt, The CP-Violating 2HDM in Light of a Strong First Order Electroweak Phase Transition and Implications for Higgs Pair Production, JHEP 03 (2018) 061 [arXiv: 1711.04097] [INSPIRE].

[19] P. Basler and M. Mühlleitner, BSMPT (Beyond the Standard Model Phase Transitions): A tool for the electroweak phase transition in extended Higgs sectors, Comput. Phys. Commun. 237 (2019) 62 [arXiv: 1803.02846] [INSPIRE].

[20] J.E. Camargo-Molina, B. O'Leary, W. Porod and F. Staub, Vevacious: A Tool For Finding The Global Minima Of One-Loop Effective Potentials With Many Scalars, Eur. Phys. J. C 73 (2013) 2588 [arXiv: 1307.1477] [INSPIRE].

[21] J.E. Camargo-Molina, B. Garbrecht, B. O'Leary, W. Porod and F. Staub, Constraining the Natural MSSM through tunneling to color-breaking vacua at zero and non-zero temperature, Phys. Lett. B 737 (2014) 156 [arXiv:1405.7376] [INSPIRE].

[22] W.G. Hollik, G. Weiglein and J. Wittbrodt, Impact of Vacuum Stability Constraints on the Phenomenology of Supersymmetric Models, JHEP 03 (2019) 109 [arXiv:1812.04644] [INSPIRE]. 
[23] A. Andreassen, D. Farhi, W. Frost and M.D. Schwartz, Direct Approach to Quantum Tunneling, Phys. Rev. Lett. 117 (2016) 231601 [arXiv:1602.01102] [INSPIRE].

[24] A. Andreassen, D. Farhi, W. Frost and M.D. Schwartz, Precision decay rate calculations in quantum field theory, Phys. Rev. D 95 (2017) 085011 [arXiv:1604.06090] [InSPIRE].

[25] M. Muhlleitner, M.O.P. Sampaio, R. Santos and J. Wittbrodt, The N2HDM under Theoretical and Experimental Scrutiny, JHEP 03 (2017) 094 [arXiv: 1612.01309] [INSPIRE].

[26] J. Velhinho, R. Santos and A. Barroso, Tree level vacuum stability in two Higgs doublet models, Phys. Lett. B 322 (1994) 213 [inSPIRE].

[27] C.C. Nishi, CP violation conditions in N-Higgs-doublet potentials, Phys. Rev. D 74 (2006) 036003 [Erratum ibid. D 76 (2007) 119901] [hep-ph/0605153] [INSPIRE].

[28] M. Maniatis, A. von Manteuffel, O. Nachtmann and F. Nagel, Stability and symmetry breaking in the general two-Higgs-doublet model, Eur. Phys. J. C 48 (2006) 805 [hep-ph/0605184] [INSPIRE].

[29] C.C. Nishi, The Structure of potentials with N Higgs doublets, Phys. Rev. D 76 (2007) 055013 [arXiv: 0706 . 2685] [INSPIRE].

[30] M. Maniatis, A. von Manteuffel and O. Nachtmann, $C P$ violation in the general two-Higgs-doublet model: A Geometric view, Eur. Phys. J. C 57 (2008) 719 [arXiv: 0707.3344] [INSPIRE].

[31] M. Maniatis, A. von Manteuffel and O. Nachtmann, A New type of CP symmetry, family replication and fermion mass hierarchies, Eur. Phys. J. C 57 (2008) 739 [arXiv:0711.3760] [INSPIRE].

[32] C.C. Nishi, Physical parameters and basis transformations in the Two-Higgs-Doublet model, Phys. Rev. D 77 (2008) 055009 [arXiv:0712.4260] [INSPIRE].

[33] M. Maniatis and O. Nachtmann, On the phenomenology of a two-Higgs-doublet model with maximal CP symmetry at the LHC, JHEP 05 (2009) 028 [arXiv:0901.4341] [INSPIRE].

[34] P.M. Ferreira, M. Maniatis, O. Nachtmann and J.P. Silva, CP properties of symmetry-constrained two-Higgs-doublet models, JHEP 08 (2010) 125 [arXiv:1004.3207] [INSPIRE].

[35] I.P. Ivanov and C.C. Nishi, Properties of the general NHDM. I. The Orbit space, Phys. Rev. D 82 (2010) 015014 [arXiv: 1004.1799] [InSPIRE].

[36] I.P. Ivanov and C.C. Nishi, Symmetry breaking patterns in 3HDM, JHEP 01 (2015) 021 [arXiv: 1410.6139] [INSPIRE].

[37] P.M. Ferreira, The vacuum structure of the Higgs complex singlet-doublet model, Phys. Rev. D 94 (2016) 096011 [arXiv: 1607.06101] [INSPIRE].

[38] A. Barroso, P.M. Ferreira, R. Santos and J.P. Silva, Stability of the normal vacuum in multi-Higgs-doublet models, Phys. Rev. D 74 (2006) 085016 [hep-ph/0608282] [INSPIRE].

[39] R. Coimbra, M.O.P. Sampaio and R. Santos, ScannerS: Constraining the phase diagram of a complex scalar singlet at the LHC, Eur. Phys. J. C 73 (2013) 2428 [arXiv:1301.2599] [INSPIRE].

[40] P.M. Ferreira, R. Guedes, M.O.P. Sampaio and R. Santos, Wrong sign and symmetric limits and non-decoupling in 2HDMs, JHEP 12 (2014) 067 [arXiv:1409.6723] [INSPIRE]. 
[41] R. Costa, M. Mühlleitner, M.O.P. Sampaio and R. Santos, Singlet Extensions of the Standard Model at LHC Run 2: Benchmarks and Comparison with the NMSSM, JHEP 06 (2016) 034 [arXiv:1512.05355] [INSPIRE].

[42] K.G. Klimenko, On Necessary and Sufficient Conditions for Some Higgs Potentials to Be Bounded From Below, Theor. Math. Phys. 62 (1985) 58 [INSPIRE].

[43] M. Mühlleitner, M.O.P. Sampaio, R. Santos and J. Wittbrodt, Phenomenological Comparison of Models with Extended Higgs Sectors, JHEP 08 (2017) 132 [arXiv:1703.07750] [INSPIRE].

[44] J. Haller, A. Hoecker, R. Kogler, K. Mönig, T. Peiffer and J. Stelzer, Update of the global electroweak fit and constraints on two-Higgs-doublet models, Eur. Phys. J. C 78 (2018) 675 [arXiv: 1803.01853] [INSPIRE].

[45] W. Grimus, L. Lavoura, O.M. Ogreid and P. Osland, A Precision constraint on multi-Higgs-doublet models, J. Phys. G 35 (2008) 075001 [arXiv:0711.4022] [INSPIRE].

[46] W. Grimus, L. Lavoura, O.M. Ogreid and P. Osland, The Oblique parameters in multi-Higgs-doublet models, Nucl. Phys. B 801 (2008) 81 [arXiv:0802.4353] [INSPIRE].

[47] P. Bechtle, O. Brein, S. Heinemeyer, G. Weiglein and K.E. Williams, HiggsBounds: Confronting Arbitrary Higgs Sectors with Exclusion Bounds from LEP and the Tevatron, Comput. Phys. Commun. 181 (2010) 138 [arXiv:0811.4169] [INSPIRE].

[48] P. Bechtle, O. Brein, S. Heinemeyer, G. Weiglein and K.E. Williams, HiggsBounds 2.0.0: Confronting Neutral and Charged Higgs Sector Predictions with Exclusion Bounds from LEP and the Tevatron, Comput. Phys. Commun. 182 (2011) 2605 [arXiv:1102.1898] [InSPIRE].

[49] P. Bechtle et al., Recent Developments in HiggsBounds and a Preview of HiggsSignals, PoS (CHARGED2012) 024 [arXiv: 1301.2345] [INSPIRE].

[50] P. Bechtle et al., HiggsBounds - 4: Improved Tests of Extended Higgs Sectors against Exclusion Bounds from LEP, the Tevatron and the LHC, Eur. Phys. J. C 74 (2014) 2693 [arXiv:1311.0055] [INSPIRE].

[51] P. Bechtle, S. Heinemeyer, O. Stål, T. Stefaniak and G. Weiglein, Applying Exclusion Likelihoods from LHC Searches to Extended Higgs Sectors, Eur. Phys. J. C 75 (2015) 421 [arXiv: 1507.06706] [INSPIRE].

[52] O. Stål and T. Stefaniak, Constraining extended Higgs sectors with HiggsSignals, PoS (EPS-HEP2013) 314 [arXiv: 1310.4039] [INSPIRE].

[53] P. Bechtle, S. Heinemeyer, O. Stål, T. Stefaniak and G. Weiglein, HiggsSignals: Confronting arbitrary Higgs sectors with measurements at the Tevatron and the LHC, Eur. Phys. J. C 74 (2014) 2711 [arXiv: 1305.1933] [InSPIRE].

[54] P. Bechtle, S. Heinemeyer, O. Stål, T. Stefaniak and G. Weiglein, Probing the Standard Model with Higgs signal rates from the Tevatron, the LHC and a future ILC, JHEP 11 (2014) 039 [arXiv:1403.1582] [INSPIRE].

[55] I. Engeln, M. Mühlleitner and J. Wittbrodt, N2HDECAY: Higgs Boson Decays in the Different Phases of the N2HDM, Comput. Phys. Commun. 234 (2019) 256 [arXiv: 1805.00966] [INSPIRE].

[56] R.V. Harlander, S. Liebler and H. Mantler, SusHi: A program for the calculation of Higgs production in gluon fusion and bottom-quark annihilation in the Standard Model and the MSSM, Comput. Phys. Commun. 184 (2013) 1605 [arXiv:1212.3249] [INSPIRE]. 
[57] R.V. Harlander, S. Liebler and H. Mantler, SusHi Bento: Beyond NNLO and the heavy-top limit, Comput. Phys. Commun. 212 (2017) 239 [arXiv:1605.03190] [INSPIRE].

[58] A. Morgan, Solving polynomial systems using continuation for engineering and scientific problems, Prentice-Hall, Upper Saddle River U.S.A. (1987).

[59] M. Maniatis and D. Mehta, Minimizing Higgs Potentials via Numerical Polynomial Homotopy Continuation, Eur. Phys. J. Plus 127 (2012) 91 [arXiv:1203.0409] [InSPIRE].

[60] S.R. Coleman, The Fate of the False Vacuum. 1. Semiclassical Theory, Phys. Rev. D 15 (1977) 2929 [Erratum ibid. D 16 (1977) 1248] [INSPIRE].

[61] C.G. Callan Jr. and S.R. Coleman, The Fate of the False Vacuum. 2. First Quantum Corrections, Phys. Rev. D 16 (1977) 1762 [InSPIRE].

[62] F.C. Adams, General solutions for tunneling of scalar fields with quartic potentials, Phys. Rev. D 48 (1993) 2800 [hep-ph/9302321] [INSPIRE].

[63] A. Masoumi, K.D. Olum and J.M. Wachter, Approximating tunneling rates in multi-dimensional field spaces, JCAP 10 (2017) 022 [arXiv:1702.00356] [INSPIRE]. 